NUB-3263

YITP-SB-09-10

\title{
Gluino NLSP, Dark Matter via Gluino Coannihilation, and LHC Signatures
}

\author{
Daniel Feldman ${ }^{1}$, Zuowei $\mathrm{Liu}^{2}$, and Pran $\mathrm{Nath}^{1}$ \\ ${ }^{1}$ Department of Physics, Northeastern University, Boston, MA 02115, USA, \\ ${ }^{2}$ C. N. Yang Institute for Theoretical Physics, \\ Stony Brook University, Stony Brook, NY 11794, USA.
}

\begin{abstract}
The possibility that the gluino is the next to the lightest supersymmetric particle (NLSP) is discussed and it is shown that this situation arises in nonuniversal SUGRA models within a significant part of the parameter space compatible with all known experimental bounds. It is then shown that the gluino NLSP (GNLSP) models lead to a compressed sfermion spectrum with the sleptons often heavier than the squarks at least for the first two generations. The relic density here is governed by gluino coannihilation which is responsible for a relatively small mass splitting between the gluino and the neutralino masses. Thus the GNLSP class of models is very predictive first because the SUSY production cross sections at the LHC are dominated by gluino production and second because the gluino production itself proceeds dominantly through a single channel which allows for a direct determination of the gluino mass and an indirect determination of the neutralino mass due to a linear relation between these two masses which is highly constrained by coannihilation. A detailed analysis of these models shows that the jet production and tagged b-jets from the gluino production can be discriminated from the standard model background with appropriate cuts. It is found that the GNLSP models can be tested with just $10 \mathrm{fb}^{-1}$ of integrated luminosity and may therefore be checked with low luminosity runs in the first data at the LHC. Thus if a GNLSP model is realized, the LHC will turn into a gluino factory through a profuse production of gluinos with typically only a small fraction $\lesssim 5 \%$ of total SUSY events arising from other production modes over the allowed GNLSP model parameter space.
\end{abstract}




\section{Introduction}

One of the interesting possibilities that arises within the landscape of possible sparticle mass hierarchies [1] is that the gluino $(\tilde{g})$ is the next to the lightest supersymmetric particle (NLSP) where neutralino dark matter produces the correct relic abundance of such matter consistent with the WMAP observations [2]. In fact, an analysis in the context of nonuniversal supergravity models (NUSUGRA) reveals that the gluino NLSP model (GNLSP) arises in a significant part of the parameter space [3, 4]. Amongst the various possible ways that the first four lightest sparticles may stack up in their mass hierarchy, one finds three such hierarchical mass patterns where the gluino is the NLSP [3, 4] which have been classified as models (NUSP13, NUSP14, NUSP15) 1 as given in Table(11). We will often refer to this subclass of NUSUGRA as the GNLSP class of models. Although progress has been made on the parameter space of sparticle masses with coannhilating gluinos [5, 6, 3, 4, 7, collider and dark matter detection implications of the GNLSP models have yet to be explored in any great detail. Thus in this work we give a dedicated analysis of such a model. Since the gluino is a strongly interacting particle, an NLSP gluino will change drastically the typical sparticle analyses. The phenomenology of GNLSP models is very different from that of a model where the gluino is the LSP [8] which we do not discuss in this paper. We note also that while relatively light gluinos have been studied in detail in reduced $S U(3)$ gaugino mass models [9], the GNLSP situation, which we cover here, was not explored.

\begin{tabular}{c|l} 
NUSP & Mass Pattern \\
\hline NUSP13 & $\widetilde{\chi}^{0}<\tilde{g}<\widetilde{\chi}_{1}^{ \pm} \lesssim \widetilde{\chi}_{2}^{0}$ \\
NUSP14 & $\widetilde{\chi}^{0}<\tilde{g}<\widetilde{t}_{1}<\widetilde{\chi}_{1}^{ \pm}$ \\
NUSP15 & $\widetilde{\chi}^{0}<\tilde{g}<A \sim H$
\end{tabular}

Table 1: Hierarchical sparticle mass patterns for the four lightest sparticles, where $\tilde{\chi}^{0} \equiv \tilde{\chi}_{1}^{0}$ is the LSP neutralino, and where the gluino is the NLSP that arises in the NUSUGRA models. The labeling of the mass patterns is as given in [3, 4].

The outline of the rest of the paper is as follows: In Sec.(2) we discuss the origin of nonuniversalities in the gaugino masses in $S U(5), S O(10)$ and $E_{6}$ GUT models. Thus this means that the ratio of the $U(1), S U(2)$ and $S U(3)$ gaugino masses at the grand unification scale $M_{G}$ are not in the ratio $1: 1: 1$. Here we point out that while no $F$ term breaking with a single irreducible representation can generate a GNLSP model, it is possible to do so with a mixture of two (or more) such breakings. Specifically we consider a linear combination of breaking with a singlet and a non-singlet $F$ term and show that several models exist which lead to a GNLSP model. We also show that there exists a subclass of models which superficially look different but are in fact isomorphic. In Sec.(3) we discuss the techniques for the computation of the sparticle spectrum at

\footnotetext{
${ }^{1}$ There is another sparticle mass pattern NUSP10 4] with the mass hierarchy $\widetilde{\chi}^{0}<\widetilde{t}_{1}<\tilde{g}<\tilde{\chi}_{1}^{ \pm}$, where $\widetilde{t}_{1}$ is the NLSP, but the $\tilde{g}$ lies close to the $\widetilde{t}_{1}$ mass.
} 
the weak scale and also discuss the experimental constraints that are imposed on the spectrum. In Sec.(4) we give a discussion of how the relic density consistent with WMAP data is satisfied under the assumption that dark matter is entirely constituted of cold dark matter in the form of $\mathrm{R}$ parity odd LSP neutralinos. Here it is shown that there are two main mechanisms by which this can come about. The first mechanism is by coannihilation with gluinos where the dominant processes which participate in the coannihilation are $\tilde{\chi}^{0} \tilde{\chi}^{0} \rightarrow f \bar{f}, \tilde{\chi}^{0} \tilde{g} \rightarrow q \bar{q}, \tilde{g} \tilde{g} \rightarrow g g, q \bar{q}$. The second mechanism is the one where the LSP has a significant higgsino component and here the relic density constraint is satisfied in a similar fashion as in the usual higgsino dominated LSP model. However, this is rather rare, and when it occurs, it is often with a small amount of gluino coannihilation. In Sec.(5) we delineate the allowed parameter space for the GNLSP models and show that there is a significant region of the parameter space where such models manifest. In Sec.(66) we show that the GNLSP models lead to a compressed sfermion spectrum for the first two generations. Specifically the sleptons and the squarks of the first two generations are essentially degenerate with the sleptons sometimes being heavier than the squarks. In Sec.(7) we give an analysis of the signatures for GNLSP models. Here we discuss three sets of post trigger level cuts labeled $\mathrm{C} 1, \mathrm{C} 2$ and $\mathrm{C} 3$ which are designed to reduce the background and enhance the signal to background ratio. It is found that the dominant signatures are jets and missing energy and with properly chosen post trigger level cuts they stand out above the background. It is further found that a discovery of a GNLSP model can come about with an integrated luminosity of $10 \mathrm{fb}^{-1}$ at the LHC with a gluino mass of up to $800 \mathrm{GeV}$. More generally, the models discussed here can be put to test with the first data from the LHC.

In Sec.(8) we discuss the direct detection of dark matter in GNLSP models. It is found that the CDMS-08 data already constrains the parameter space of GNLSP models, although only rather mildly at the level of $\sigma_{S I}\left(\tilde{\chi}^{0} p\right) \simeq 10^{-44} \mathrm{~cm}^{2}$. Further, the future data from CDMS and LUX will either detect dark matter predicted in this model or constrain the parameter space of the model. It is also noted, however, that a part of the parameter space of the model leads to a rather small spin independent neutralino-proton cross sections, i.e., $\sigma_{S I}\left(\tilde{\chi}^{0} p\right)<10^{-46} \mathrm{~cm}^{2}$, which lies outside the reach of the current direct detection experiments and similar experiments in the foreseeable future. Interestingly, much of this parameter space will be accessible at the LHC since the gluinos can be produced and detected via their jet and missing energy signatures as discussed in Sec.(7). In this sense, the LHC and the direct detection experiments are complementary. In Sec.(9) we discuss the benchmarks for the three GNLSP model sets A, B and C.

Conclusions are given in Sec.(10). In Appendix A we give sum rules on the gaugino masses that hold for the various cases of nonuniversalities that appear in Table (2). These sum rules also hold when one includes a singlet breaking along with breaking with a non-singlet. In Appendix $\mathrm{B}$ we give an analysis at one loop which explains the compression of the sfermion spectrum for the first two generations. Benchmarks discussed in Sec.(9) are given in Appendix C. 


\section{Gaugino mass nonuniversalities in GUT models, the gluino NLSP and scaling}

There is considerable literature on nonuniversalities of soft breaking and their applications [10] within the framework of supergravity grand unification[11, 12. Our focus will be on the gluino phenomenology that results from the gluino being an NLSP [For recent analyses related to gluino phenomenology in various contexts see [13]]. Specifically, our focus here will be on nonuniversalities in the gaugino mass sector arising from $F$ type breaking in $S U(5), S O(10)$, and $E_{6}$ GUT groups which have been discussed over the years [14, 15] and a more comprehensive analysis has been given recently[16]. Results of this analysis are summarized in Table(21) 2. In the table, ratios of gaugino masses that arise when the GUT symmetry is broken by an $F$ term, which is an irreducible representation of the gauge group $S U(5), S O(10)$, and $E_{6}$, and enters in the decomposition of the symmetric product of two adjoint representations corresponding to the relevant group. Table(21) identifies the group and the irreducible representation and the corresponding ratio of the gaugino masses. For $S O(10)$ and $E_{6}$ several gaugino mass ratios are listed for a given irreducible representation. These correspond to different patterns by which the GUT symmetry breaks to lower rank groups. Further details can be found in [16]. None of the models listed in

\begin{tabular}{|c|ccc|c|ccc|}
\hline Group & Rep. & Label & $M_{1}: M_{2}: M_{3}$ & Group & Rep. & Label & $M_{1}: M_{2}: M_{3}$ \\
\hline$S U(5)$ & $\mathbf{1}$ & - & $1: 1: 1$ & $E_{6}$ & $\mathbf{6 5 0}$ & $(12)$ & $-1: 1: 1$ \\
& $\mathbf{2 4}$ & $(1)$ & $-1 / 2:-3 / 2: 1$ & & & $(13)$ & $-1: 1: 0$ \\
& $\mathbf{7 5}$ & $(2)$ & $-5: 3: 1$ & & & $(14)$ & $1 / 10:-3 / 2: 1$ \\
& $\mathbf{2 0 0}$ & $(3)$ & $10: 2: 1$ & & & $(15)$ & $-13 / 5: 1: 1$ \\
$S O(10)$ & $\mathbf{2 1 0}$ & $(4)$ & $-3 / 5: 1: 0$ & & & $(16)$ & $1 / 5: 1: 0$ \\
& & $(5)$ & $-4 / 5: 0: 1$ & & & $(17)$ & $41 / 15: 1: 1$ \\
& & $(6)$ & $1: 0: 0$ & & $\mathbf{2 4 3 0}$ & $(18)$ & $-11 / 5: 1: 0$ \\
& $\mathbf{7 7 0}$ & $(7)$ & $19 / 10: 5 / 2: 1$ & & & $(19)$ & $1: 35 / 9: 1$ \\
& & $(8)$ & $32 / 5: 0: 0$ & & & $(20)$ & $12 / 5: 0: 1$ \\
$E_{6}$ & $\mathbf{6 5 0}$ & $(9)$ & $-1 / 5: 1: 0$ & & & $(21)$ & $0: 0: 1$ \\
& & $(10)$ & $-1 / 5:-1: 1$ & & & $(22)$ & $33 / 5: 1: 1$ \\
& & $(11)$ & $3: 1: 1$ & & & $(23)$ & $9 / 5: 1: 0$ \\
\hline
\end{tabular}

Table 2: Exhibition of the gaugino mass ratios at the GUT scale for various groups and representations in $S U(5), S O(10)$, and $E_{6}$ models [16]. The mass ratios are listed in a hierarchical manner, i.e., they are listed in the order of the smallest rank group and lowest dimensional representation in which they first appear and are labeled from (1)-(23). Thus a specific ratio may be repeated several times as one goes up the chain.

Table(2) can give rise to a gluino as the NLSP with $F$ type breaking with a single irreducible

\footnotetext{
${ }^{2}$ In this analysis we do not consider the flipped models and the ratios listed in Table (2) exclude such models.
} 
representation 3 . However, we will show that a combination of GUT symmetry breaking in the gaugino mass term sector with two irreducible representations does allow for a gluino as the NLSP for a subset of models listed in Table(2). Specifically we will consider a linear combination of a singlet and a non-singlet $F$ term. In this case an interesting phenomenon arises in that the models with the same value $r \equiv\left(M_{2}-M_{1}\right) /\left(M_{3}-M_{1}\right)$ can be made isomorphic under redefinitions and scalings in the gaugino sector. Thus suppose we write the gaugino masses for models of the above type with a singlet and a non-singlet $\mathrm{F}$ breaking so that

$$
M_{1}^{(i)}=\left(1+a_{i} \alpha_{i}\right) m_{1 / 2}, \quad M_{2}^{(i)}=\left(1+b_{i} \alpha_{i}\right) m_{1 / 2}, M_{3}^{(i)}=\left(1+c_{i} \alpha_{i}\right) m_{1 / 2},
$$

where the first term within each of the parentheses on the right hand side in Eq.(1) arises from the singlet contribution, and the second term within each of the parentheses is the contribution from the non-singlet. Here $i$ defines a specific model and $a_{i}, b_{i}, c_{i}$ are the fractions given in Table(2) with $\alpha_{i}$ being an arbitrary parameter. Next we note that two models $i$ and $j$ defined by Eq.(1) can be made isomorphic if they have the same value of $r$ in the sense that

$$
M_{a}^{(i)}=\lambda_{i j} M_{a}^{(j)} ; a=1,2,3,
$$

when $\alpha_{j}$ is related to $\alpha_{i}$ in the following way

$$
\alpha_{j}^{-1}\left(b_{i}-a_{i}\right)=\alpha_{i}^{-1}\left(b_{j}-a_{j}\right)+a_{i} b_{j}-b_{i} a_{j} .
$$

This means that under the constraint of Eq.(3) , a rescaling of $m_{1 / 2}$ of model $j$ can make it isomorphic to model $i$. Thus in essence, models with the same value of $r$ would in fact be equivalent when taken in a linear combination of breakings including singlets. Using Eq.(1) and Table(2) one finds that there are several possibilities for which the GNLSP class of models can arise. We limit ourselves to the following cases:

1. Model GNLSP A (ISO-I) : This class of models arise where $r$ takes the common value $-2 / 3$ as exhibited below

$$
\left.\begin{array}{c}
M_{1}: M_{2}: M_{3} \\
\hline-1 / 2:-3 / 2: 1 \\
19 / 10: 5 / 2: 1 \\
-1 / 5:-1: 1
\end{array}\right\} \longrightarrow r=-2 / 3 \text {. }
$$

2. Model GNLSP $\mathrm{B}_{\mathrm{B}}$ : This is an $E_{6}$ model with $F$ type breaking with 2430 plet such that [16] $E_{6} \rightarrow S U(6)^{\prime \prime} \times S U(2)_{L}(2430 \rightarrow(189,1))$ which gives $M_{1}: M_{2}: M_{3}=0: 0: 1$. This model can generate a gluino as the NLSP upon the addition of breaking with a singlet 4 .

\footnotetext{
${ }^{3}$ This also holds for flipped models, i.e., $F$ type breaking with a single irreducible representation cannot give rise to a gluino as the NLSP.

${ }^{4}$ We note that there is another $E_{6}$ model with $F$ type breaking with 2430 plet such that $E_{6} \rightarrow S U(6)^{\prime \prime} \times$ $S U(2)_{L}(2430 \rightarrow(405,1))\left[16\right.$ ] which gives $M_{1}: M_{2}: M_{3}=\frac{12}{5}: 0: 1(r=12 / 7)$. This model can also generate a gluino as the NLSP upon addition of a singlet and there is a relative sign flip between $M_{1}$ and $M_{2}$ in this case. However, the model gives a light Higgs mass in the parameter space investigated which falls below the current limits and thus we do not consider this model further.
} 
3. Model GNLSP $\mathrm{C}_{\mathrm{C}}$ : Here $r$ is free and thus defining $r=\delta_{2} / \delta_{3}$ the gaugino masses at the GUT scale may be parametrized as

$$
\widetilde{M}_{1}=m_{1 / 2}, \quad \widetilde{M}_{2}=\left(1+\delta_{2}\right) m_{1 / 2}, \quad \widetilde{M}_{3}=\left(1+\delta_{3}\right) m_{1 / 2},
$$

and $\delta_{2}$ and $\delta_{3}$ can be varied independently. Model GNLSP $\mathrm{C}_{\mathrm{C}}$ contains models GNLSP $A$ and GNLSP $_{\mathrm{B}}$ as subcase: 5 .

Aside from the model discussed in footnote 4, models GNLSP $\mathrm{A}, \mathrm{GNLSP}_{\mathrm{B}}$, and GNLSP $_{\mathrm{C}}$ are the only models which lead to a GNLSP through breaking with a singlet and a nonsinglet. This can be seen easily by using the semi analytic analysis given in Appendix B. For all the three models a GNLSP requires $\delta_{3}$ to lie in the range $(-0.9,-0.8)$. Some benchmarks for Models A,B and $\mathrm{C}$ are given in Tables (618]10) and a display of their partial sparticle spectrum and some other properties of these models are exhibited in Tables(7],9]11). We also note that from the analysis of [16] one can discern another set of models which have the same common value of $r$. Thus the models with the gaugino mass ratios $M_{1}: M_{2}: M_{3}=$ (i) $-\frac{1}{5}: 3: 1$; (ii) $\frac{2}{5}: 2: 1$; (iii) $-\frac{3}{5}: 1: 0$;(iv) $\frac{5}{2}:-\frac{3}{2}: 1 ;$ (v) $\frac{1}{10}: \frac{5}{2}: 1 ;$ (vi) $\frac{8}{5}: 0: 1$ have the common value $r=8 / 3$. One may call this ISO-II because when combined with a singlet $F$ type breaking these models too would be isomorphic so that the six different models are effectively one model as far as the gaugino sector is concerned. However, this model class does not lead to a GNLSP which is the main focus of this paper. In the following we discuss the GNLSP models in further detail including the satisfaction of the relic density, the production cross section of the gluinos, the signatures for their identification at the LHC, and the direct detection of dark matter in the GNLSP class of models.

\section{$3 \quad$ Experimental constraints}

Our general procedure is similar to that discussed in [4 which we briefly describe below. In the analysis one specifies boundary conditions of the model at the grand unification (GUT) scale which we take to be $M_{G} \sim 2 \times 10^{16} \mathrm{GeV}$. Specifically we take the sfermion masses at the GUT scale to be universal, but assume that the gaugino masses are in general nonuniversal with nonuniversalities given by $\delta_{2}$ and $\delta_{3}$. One then uses renormalization group equations (RGEs) to compute the sparticle mass matrices and their eigenvalues at the electroweak scale. The code used in these RGE evolutions and computations of the sparticle spectrum is SuSpect2.41 [17], and similar results are obtained with SoftSUSY[18] and SPheno [19]. Further, one imposes the lower limit constraints on the sparticle masses from the LEP and from the Tevatron data as well as constraints from the WMAP on the relic density. The analysis of the relic density is first done at the perturbative level with MicrOMEGAs [20, which relies on CalcHEP [21]. Non-perturbative effects on the relic density are also discussed.

\footnotetext{
${ }^{5}$ We remark that in [3, 4, where the gluino NLSP in SUGRA models was previously observed, the notation $\delta_{5}, \delta_{6}$ was used.
} 
Below we give a list of the relevant constraints from collider and astrophysical data which have been included in the analysis:

(i) The 5-year WMAP data constrains the relic density of dark matter in the universe so that $\Omega_{\mathrm{DM}} h^{2}=0.1131 \pm 0.0034$ [22]. We take a $6 \sigma$ corridor around the central value to constrain the relic abundance of neutralinos. The larger band is taken due to the sensitivity of the relic density computation in particular regions of the parameter space. A large class of our models fall well within a $2 \sigma$ bound.

(ii) The FCNC process $b \rightarrow s \gamma$ receives a significant contribution from the SUSY processes[23]. The Heavy Flavor Averaging Group (HFAG) 24] along with the BABAR, Belle and CLEO give experimental results: $\mathcal{B} r\left(B \rightarrow X_{s} \gamma\right)=(352 \pm 23 \pm 9) \times 10^{-6}$. A new estimate of standard model contributions at $O\left(\alpha_{s}^{2}\right)$ gives [25] $\mathcal{B} r(b \rightarrow s \gamma)=(3.15 \pm 0.23) \times 10^{-4}$. We utilize both experimental and theoretical progress in the evaluation of this observable and take a $3 \sigma$ corridor around the experimental value, $2.77 \times 10^{-4}<\mathcal{B} r(b \rightarrow s \gamma)<4.27 \times 10^{-4}$, to constrain the theoretical prediction including both SM and SUSY contributions.

(iii) Another important constraint from B-physics is the rare decay process $B_{s} \rightarrow \mu^{+} \mu^{-}$ which can become significant for large $\tan \beta[26$. The most stringent 95\% (90\%) C.L. limits are achieved by $\operatorname{CDF}$ [27] $\mathcal{B} r\left(B_{s} \rightarrow \mu^{+} \mu^{-}\right)<5.8 \times 10^{-8}\left(4.7 \times 10^{-8}\right)$. We take a conservative limit $\mathcal{B} r\left(B_{s} \rightarrow \mu^{+} \mu^{-}\right)<10^{-7}$.

(iv) For the constraints from the anomalous magnetic moment of the muon, we use a conservative bound $-11.4 \times 10^{-10}<\delta\left(g_{\mu}-2\right)<9.4 \times 10^{-9}$ as in [28] where $\delta\left(g_{\mu}-2\right)$ is the new physics contribution to $\left(g_{\mu}-2\right)$ beyond the standard model.

(v) Additionally, we also impose various mass limits as follows: $m_{\widetilde{\chi}_{1}^{ \pm}}>104.5 \mathrm{GeV}$ [29] for the lighter chargino, $m_{\widetilde{t}_{1}}>101.5 \mathrm{GeV}$ for the lighter stop, and $m_{\widetilde{\tau}_{1}}>98.8 \mathrm{GeV}$ for the lighter stau. For the lightest CP even Higgs boson mass in MSSM we take the constraint to be $m_{h}>100 \mathrm{GeV}$ ( $90 \%$ of the models that pass all constraints have $m_{h}>110 \mathrm{GeV}$ ). One may compare these with the standard model like Higgs boson mass limit which is $\approx 114.4 \mathrm{GeV}[30$. For the gluino mass, recent Tevatron experiments give $m_{\tilde{g}}>308 \mathrm{GeV}$ (D-Zero) [31] and $m_{\tilde{g}}>280 \mathrm{GeV}$ (CDF) [32]. The limits given by [31, 32] are valid within the framework of the minimal supergravity models and may be modified in nonuniversal SUGRA models. Hence the total SUSY production cross section constrained by the Tevatron analyses will typically be a larger total cross section than that which arises in the GNLSP models. Further, as we will show shortly, the mass splitting between the NLSP gluino and LSP neutralino must be relatively small in order to satisfy relic density constraints. Thus the relatively small mass splittings between the LSP and GNSLP can lead to softer decay products and an overall lower multiplicity of final state events relative to models for which the mass splitting is significantly larger. Therefore in this analysis, we take a conservative lower bound, namely $m_{\tilde{g}}>220 \mathrm{GeV}$. Our choice of this lower bound is taken as to not eliminate a part of the parameter space which may otherwise be allowed pending a 
full analysis of the Tevatron data using nonuniversalities (see also 33] for a related discussion regarding a lower bound on the mass of the gluino).

\section{Relic density via gluino coannihilation}

It is interesting to ask how the relic density constraints are satisfied in the class of models with the gluino as the NLSP as these constraints have important implications for collider phenomenology (for recent works connecting sparticle phenomenology at colliders and dark matter see [34, 35, [36, 37, 38]). As an illustration we consider the model

$$
\operatorname{GNLSP}_{\mathrm{Co}}:\left(m_{0}, m_{1 / 2}, A_{0}, \tan \beta, \delta_{2}, \delta_{3}\right)=(1450,730,2700,40,0.332,-0.839),
$$

where all masses are in $\mathrm{GeV}$ and $\operatorname{sign}(\mu)$ is taken to be positive. We will take the top mass at $170.9 \mathrm{GeV}$ throughout this work, though the analysis here does not show great sensitivity to the top mass. This model gives $\left(m_{\widetilde{\chi}^{0}}, m_{\tilde{g}}\right)=(305.1,348.6) \mathrm{GeV}$. For GNLSP $\mathrm{Co}_{\mathrm{o}}$ the channels which contribute to $1 /\left(\Omega h^{2}\right)_{\tilde{\chi}^{0}}$ more than $1 \%$ are as follows : $\tilde{g} \tilde{g} \rightarrow g g(47 \%), \tilde{g} \tilde{g} \rightarrow u \bar{u}(8 \%), \tilde{g} \tilde{g} \rightarrow$ $c \bar{c}(8 \%), \tilde{g} \tilde{g} \rightarrow d \bar{d}(8 \%), \tilde{g} \tilde{g} \rightarrow s \bar{s}(8 \%), \tilde{g} \tilde{g} \rightarrow b \bar{b}(6 \%), \tilde{g} \tilde{g} \rightarrow t \bar{t}(4 \%), \widetilde{\chi}^{0} \widetilde{\chi}^{0} \rightarrow b \bar{b}(6 \%), \tilde{\chi}^{0} \tilde{g} \rightarrow t \bar{t}(2 \%)$, $\tilde{\chi}^{0} \widetilde{\chi}^{0} \rightarrow t \bar{t}(2 \%), \widetilde{\chi}^{0} \widetilde{\chi}^{0} \rightarrow \tau^{+} \tau^{-}(1 \%)$. The relic density is $\left(\Omega h^{2}\right)_{\tilde{\chi}^{0}}=0.108$ at the perturbative level, and the model has eigen decomposition $\tilde{\chi}^{0}=0.986 \tilde{b}-0.016 \tilde{w}+0.146 \tilde{h}_{1}-0.092 \tilde{h}_{2}$ where

$\tilde{b}, \tilde{w}$, are the bino and wino components and $\tilde{h}_{1}, \tilde{h}_{2}$ are the higgsino components, and thus the model has a substantial higgsino component. This model belongs to the pattern classified as NUSP13 in [3, 4].

From the above it is clear that the gluino processes dominate the WIMP annihilation at the freezeout temperature in the early universe[5]. Further the LSP mass and the NLSP mass are close with a mass difference $\Delta_{\tilde{g} \tilde{\chi}^{0}} \equiv\left(m_{\tilde{g}}-m_{\tilde{\chi}^{0}}\right) / m_{\tilde{\chi}^{0}} \approx 0.14$. An examination of the mass splittings and the associated annihilation processes point to a strong coannihilation occurring in the model of Eq.([6). Thus consider the annihilation processes $\tilde{\chi}_{i} \tilde{\chi}_{j}$ going into the standard model particles. Here the effects of coannihilation are controlled by the Boltzmann suppression factor 39 ]

$$
\gamma_{i}=\frac{n_{i}^{\mathrm{eq}}}{n^{\mathrm{eq}}}=\frac{g_{i}\left(1+\Delta_{i}\right)^{3 / 2} e^{-\Delta_{i} x}}{\sum_{j} g_{j}\left(1+\Delta_{j}\right)^{3 / 2} e^{-\Delta_{j} x}},
$$

where $g_{i}$ are the degrees of freedom of $\chi_{i}, x=m_{1} / T$ and $\Delta_{i}=\left(m_{i}-m_{1}\right) / m_{1}$, with $m_{1}$ defined as the LSP mass. The processes which dominate the WIMP annihilation in the early universe are

$$
\tilde{\chi}^{0} \widetilde{\chi}^{0} \rightarrow F, \tilde{\chi}^{0} \tilde{g} \rightarrow F^{\prime}, \tilde{g} \tilde{g} \rightarrow F^{\prime \prime}
$$

where $F, F^{\prime}, F^{\prime \prime}$ constitute the pairs of standard model states. The relic density is controlled by the integral

$$
J_{x_{f}}=\int_{x_{f}}^{\infty} x^{-2}\left\langle\sigma_{\mathrm{eff}} v\right\rangle d x
$$


where $v$ is the relative velocity of annihilating supersymmetric particles, $\left\langle\sigma_{\text {eff }} v\right\rangle$ is the thermally averaged cross section times the relative velocity and $x_{f}$ is the freezeout temperature. The $\sigma_{\text {eff }}$ that enters the relic density can be written approximately as follows

$$
\sigma_{\mathrm{eff}} \simeq \sigma_{\tilde{g} \tilde{g}} \gamma_{\widetilde{\chi}^{0}}^{2}\left(\gamma^{2}+2 \gamma \frac{\sigma_{\widetilde{\chi}^{0} \tilde{g}}}{\sigma_{\tilde{g} \tilde{g}}}+\frac{\sigma_{\widetilde{\chi}^{0} \widetilde{\chi}^{0}}}{\sigma_{\tilde{g} \tilde{g}}}\right)
$$

where $\gamma=\gamma_{\tilde{g}} / \gamma_{\widetilde{\chi}^{0}}$ and where $\gamma_{i}$ are defined by Eq.(17) and where [8]

$$
\begin{aligned}
\sigma(\tilde{g} \tilde{g} \rightarrow g g) & =\frac{3 \pi \alpha_{s}^{2}}{16 \beta^{2} s}\left\{\log \frac{1+\beta}{1-\beta}\left[21-6 \beta^{2}-3 \beta^{4}\right]-33 \beta+17 \beta^{3}\right\} \\
\sigma(\tilde{g} \tilde{g} \rightarrow q \bar{q}) & =\frac{\pi \alpha_{s}^{2} \bar{\beta}}{16 \beta s}\left(3-\beta^{2}\right)\left(3-\bar{\beta}^{2}\right) .
\end{aligned}
$$

Here $\beta=\sqrt{1-4 m_{\tilde{g}}^{2} / s}$, and the quark mass enters Eq.(11) through $\bar{\beta}=\sqrt{1-4 m_{q}^{2} / s}$. One interesting phenomenon concerns the following: we know that the cross section for the annihilating gluinos falls with the gluino mass. On the other hand $\sigma_{\text {eff }}$ that enters the relic density analysis must be nearly constant for a wide range of gluino masses so that the relic density be satisfied. This can happen due to the presence of the coannihilation factor $\gamma^{2}$ which multiplies $\sigma_{\tilde{g} \tilde{g}}$ in Eq.(10). This is easily seen by noticing that $\Delta_{\tilde{g} \tilde{\chi}^{0}}=\left(m_{\tilde{g}}-m_{\tilde{\chi}^{0}}\right) / m_{\tilde{\chi}^{0}}$ has a dependence on the gluino mass of the form 6

$$
\Delta_{\tilde{g} \tilde{\chi}^{0}}^{0}-C \log \left(m_{\tilde{g}} / m_{\tilde{g}}^{0}\right)
$$

where $\Delta_{\tilde{g} \tilde{\chi}^{0}}^{0}=\left(m_{\tilde{g}}^{0}-m_{\tilde{\chi}^{0}}^{0}\right) / m_{\tilde{\chi}^{0}}^{0}$, and $m_{\tilde{g}}^{0}$ is a reference gluino mass and $m_{\tilde{\chi}^{0}}^{0}$ is the corresponding reference neutralino mass, and $C>0$. What one finds is that the difference $\Delta_{\tilde{g} \tilde{\chi}^{0}}$ decreases when gluino mass increases which enhances $\gamma$ and compensates for the falling cross section $\sigma_{\tilde{g} \tilde{g}}$. The above phenomenon sustains an essentially constant $J_{x_{f}}$ as the gluino mass varies allowing for a satisfaction of the relic density over a wide range of gluino masses. What the analysis implies is that $m_{\tilde{g}} / m_{\tilde{\chi}^{0}}$ tends to unity as the gluino mass increases. A numerical analysis bears this out. It is, however, interesting to note, that we also find few cases where the GNLSP emerges without significant coannihilation which occurs when the LSP has a significant higgsino component which allows for the satisfaction of the relic density constraint in a manner quite similar to what happens on the Hyperbolic Branch of REWSB [40]. Indeed, there are cases where the neutralino annihilations are seen to dominate the annihilation cross sections via $\widetilde{\chi}^{0} \widetilde{\chi}^{0} \rightarrow\left(b \bar{b}, \tau^{+} \tau^{-}\right)$with only a small contribution to the satisfaction of the relic density constraints arising from LSPGNLSP coannihilation, and in some cases coannihilation enters only at the single percent level (an example is GNLSP $_{\mathrm{C} 1}$ given in Appendix C). Another interesting example is model GNLSP A1 $_{\text {(also }}$ given in Appendix $\mathrm{C}$ ) which proceeds with annihilation contributions to relic density calculation dominantly via (62\%) $\widetilde{\chi}^{0} \widetilde{\chi}^{0} \rightarrow t \bar{t}$, and (15\%) $\widetilde{\chi}^{0} \widetilde{\chi}^{0} \rightarrow W^{+} W^{-}$, and only a small fraction (3\%) for $\tilde{g} \tilde{g} \rightarrow g g$ and the remainder coming from neutral diboson final states.

We discuss now possible nonperturbative corrections to the annihilation cross section. As shown in Refs [8, 5] nonperturbative effects on the annihilation cross section can be relevant near

\footnotetext{
${ }^{6} \mathrm{~A}$ similar relationship in a graphical form appears in the analysis of $[\underline{5}$ for a bino LSP.
} 
threshold where multiple gluon exchange, for example, can give rise to the so-called Sommerfeld enhancement factor $\mathcal{E}$. These effects may be approximated as [8]

$$
\mathcal{E}_{j}=\frac{C_{j} \pi \alpha_{s}}{\beta}\left[1-\exp \left\{-\frac{C_{j} \pi \alpha_{s}}{\beta}\right\}\right]^{-1},
$$

where $C_{j=g}=1 / 2\left(C_{j=q}=3 / 2\right)$ for $\tilde{g} \tilde{g} \rightarrow g g(\tilde{g} \tilde{g} \rightarrow q \bar{q})$ respectively, and we note that $\mathcal{E}_{j}$ enters bilinearly in Eq.(11).

Bound states can form as well if the gluino is a stable LSP. We do not consider the latter situation. However, as already discussed, for the GNLSP at the perturbative level, the dominant contribution in the annihilation cross section for most models arises from the gluino-gluino annihilation modes, and this occurs for $\Delta_{\tilde{g} \tilde{\chi}^{0}} \lesssim 0.2$. Since micrOMEGAs performs the relic density analysis using only perturbative cross section, we have carried out an independent analysis of the relic density to include the effects of the Sommerfeld enhancement. Our analysis gives results which are in agreement with the analysis of third reference of [5]. We note here that an increase in $\Delta_{\tilde{g} \widetilde{\chi}^{0}}$ in the range of $(2-3) \%$ is needed when the Sommerfeld enhancement of cross section is taken into account. Equivalently the effect of the Sommerfeld enhancement can be recast as a shift in the gluino mass for a fixed LSP mass in order to have the same relic density as for the perturbative case. Specifically, an upward shift of the gluino mass by a few GeV is needed. Thus

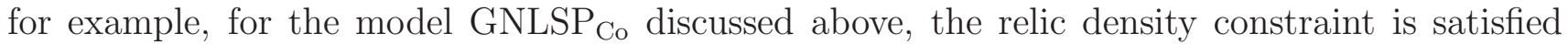
with the inclusion of non-perturbative effects with a $3 \mathrm{GeV}$ upward shift of the $S U(3)$ gaugino mass at the GUT scale leading to an increase in $\Delta_{\tilde{g} \tilde{\chi}^{0}}=14 \% \rightarrow 16 \%$. More generally, we find that numerically for the non-perturbative case, for fixed LSP mass, $m_{\tilde{g}}$ needs to be increased by (3 to 6) GeV to achieve the same relic density as for the perturbative case. The above holds for gluino masses in the range up to about $1 \mathrm{TeV}$. In Appendix $\mathrm{C}$ we give benchmarks including the effects of the Sommerfeld enhancement.

\section{Consistent parameter space of the gluino NLSP}

Based on the initial discovery of the existence of a viable parameter space where the gluino is the NLSP in nonuniversal SUGRA models [3, 4], we perform in this work a dedicated search for delineating the parameter space of GNLSP models consistent with the radiative electroweak symmetry breaking constraints and with the experimental constraints from colliders and from the relic density. In our analysis the input parameters $m_{0}, m_{1 / 2}, A_{0}, \tan \beta, \delta_{2}, \delta_{3}$ assume the following bounds: $m_{0}<4 \mathrm{TeV}, m_{1 / 2}<2 \mathrm{TeV},\left|A_{0} / m_{0}\right|<3$, and $\tan \beta \in(1,60)$, while $\delta_{2}$ and $\delta_{3}$ are chosen in a manner appropriate for models $\mathrm{A}, \mathrm{B}$ and $\mathrm{C}$ defined in Sec.(2). Thus for model GNLSP $\mathrm{A}$, $\delta_{2}$ is determined by the constraint $r=-2 / 3$, for model GNLSP G $_{2}, \delta_{2}=0$ and for the model GNLSP $_{\mathrm{C}}, \delta_{2}$ is assumed to lie in the range $\delta_{2} \in(-0.9,1)$, while $\delta_{3}$ typically lies in the range $\delta_{3} \in(-0.9,-0.8)$. Within the ranges assumed above, we find a significant region of the parameter 

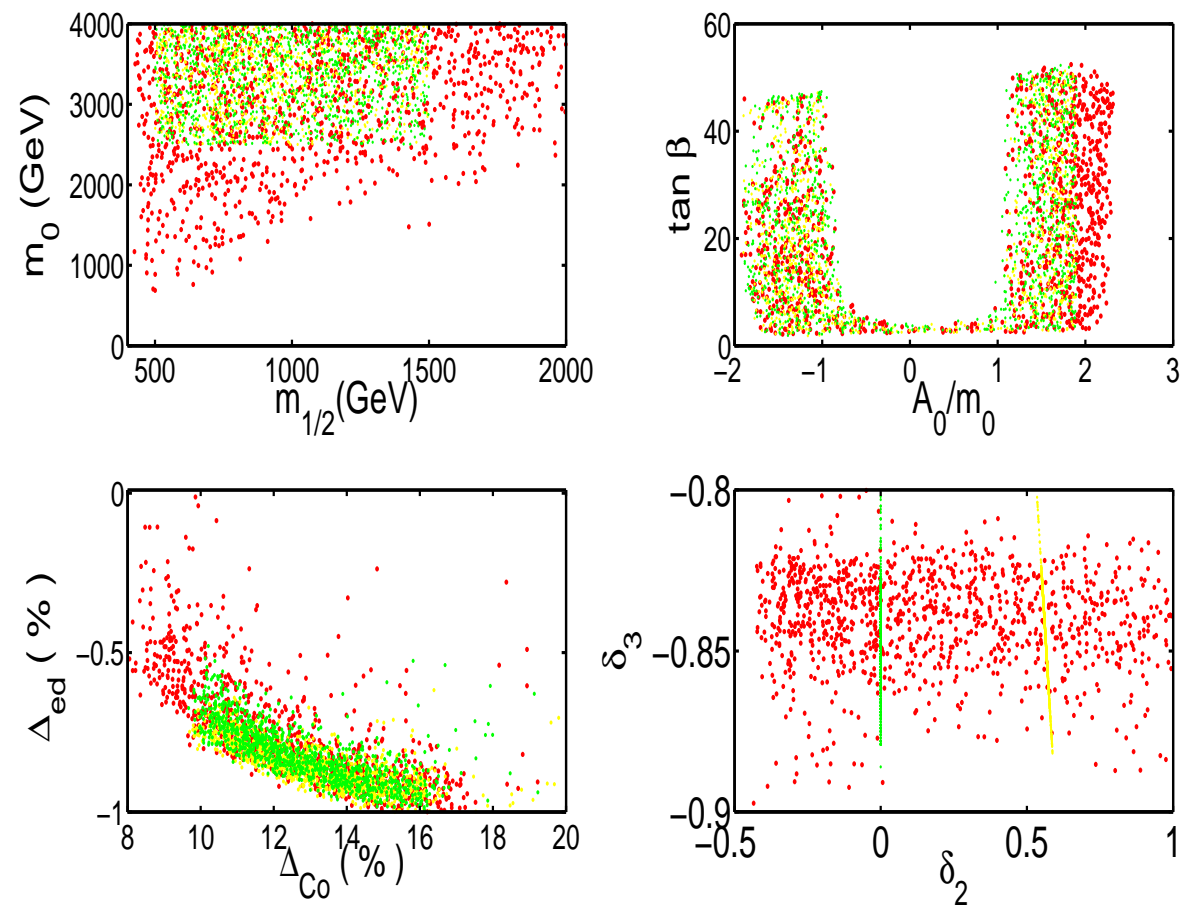

Figure 1: (Color online) An analysis of the consistent parameter space in GNLSP models. Red (dark) model points are for Model GNLSP ${ }_{\mathrm{C}}$, while the yellow (light) and the green model points are for Model GNLSP $\mathrm{A}$ and Model GNLSP G $_{\mathrm{B}}$ as discussed in the text. $\Delta_{e d}$ is the splitting of the slepton and squark masses in the first two generations as also discussed in the text.

space where each model is realized. Of course, the parameter space for model $\mathrm{GNLSP}_{\mathrm{C}}$ is larger than that for the model GNLSP $\mathrm{A}$ or for the model GNLSP G $_{\mathrm{B}}$, but the parameter space for models GNLSP A $_{\mathrm{A}}$ and GNLSP $\mathrm{B}$ are also quite significant as shown in Fig.(1). Some interesting observations can be made from the analysis of Fig.(11). Thus the top left panel of Fig. (11) shows that typically $m_{0}>m_{1 / 2}$ for this class of models while the top right panel shows that the region $A_{0} / m_{0}=0$ is very thinly populated which is in sharp contrast to the mSUGRA case where the $A_{0} / m_{0}$ region is heavily populated. The lower right panel of Fig.(1) displays the allowed model points in the $\delta_{3}$ vs $\delta_{2}$ plane which shows that GNLSP models constrain the nonuniversality $\delta_{3}$ to lie in a very narrow range $(-0.9,-0.8)\left[3\right.$, 4] while $\delta_{2}$ is widely dispersed for model GNLSP ${ }_{\mathrm{C}}$ but restricted for models GNLSP $\mathrm{A}$ and $\mathrm{GNLSP}_{\mathrm{B}}$ since $\delta_{2} / \delta_{3}=-2 / 3$ for model GNLSP $\mathrm{A}$ and $\delta_{2}=0$ for model GNLSP . These points are indicated in yellow (model GNLSP $_{\mathrm{A}}$ ) and green (model $\left.\operatorname{GNLSP}_{\mathrm{B}}\right)$. 

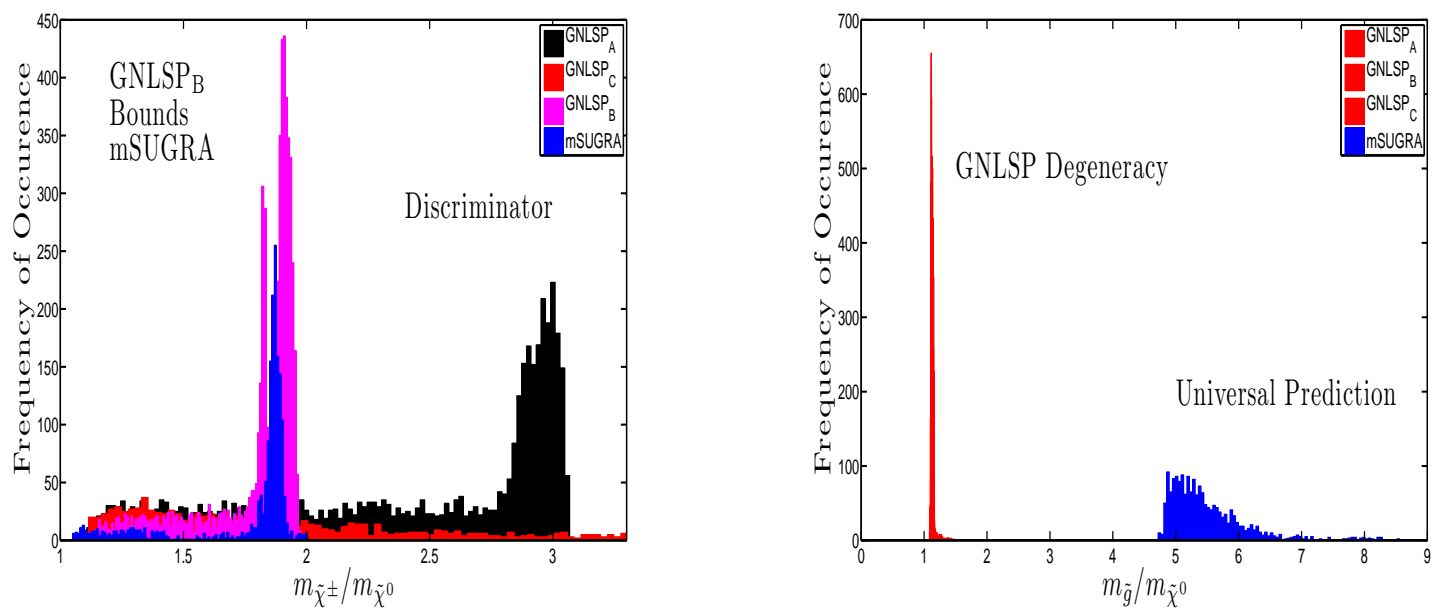

Figure 2: (Color online) Left panel: An exhibition of the scaling between the light chargino mass and the LSP mass for the GNLSP models GNLSP $_{\mathrm{A}}, \mathrm{GNLSP}_{\mathrm{B}}$ and GNLSP $_{\mathrm{C}}$ vs the mSUGRA model. The figure shows that GNLSP A produces the ratio $m_{\tilde{\chi}^{ \pm}} / m_{\tilde{\chi}^{0}} \sim 3$ which differentiates it

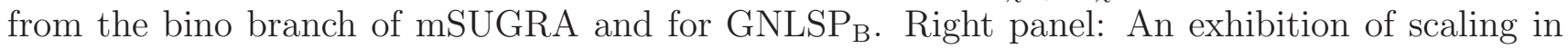
$m_{\tilde{g}} / m_{\tilde{\chi}^{0}}$. All GNLSP models are well separated from mSUGRA in this figure.

\section{Compression of the sparticle mass spectrum in GNLSP models}

In models with universal boundary conditions at the GUT scale for the gaugino masses, the gluino mass will be typically a factor of 5-6 larger than the lightest neutralino mass. A large gluino mass tends to contribute a significant portion to the squark masses in the RG running. Thus in the mSUGRA model[11] there is typically a significant splitting of the slepton and squark masses for regions of the parameter space where $m_{0}$ and $m_{1 / 2}$ are comparable. This is generally not the case in the model under consideration where gluino is the NLSP. Here the gluino mass will be typically much lighter relative to the squark masses, and thus splittings between sleptons and squarks will be less pronounced. Specifically for the first two generations one will find a rather compressed spectrum. Table(3) exhibits the high degree of degeneracy of the squarks and of the sleptons in the first two generations in models with the gluino as the NLSP relative to, e.g., the mSUGRA model [For a general discussion of sum rules see [41]]. In the examples shown one finds that while for the mSUGRA mSP37 case the splitting between the sum of the down type quarks and the charged sleptons in the first generation can be as much as $35 \%$, while for the GNLSP case it is only about 1\%. Further, while for the mSUGRA case the first and the second generation squarks are invariably heavier than their corresponding slepton counterparts, for the

\footnotetext{
${ }^{7}$ The mSUGRA pattern mSP3 has the following hierarchy for the first four sparticles: $\tilde{\chi}^{0}<\tilde{\chi}_{1}^{ \pm}<\tilde{\chi}_{2}^{0}<\tilde{\tau}_{1}$. The largeness of the sfermion masses indicates that the electroweak symmetry breaking is realized on the Hyperbolic Branch 40] (for recent works on the HB see [42, 37]) .
} 
GNLSP case one finds that one can often get an inversion, i.e., the model gives rise to sleptons heavier than their squark counterparts within the first and second generations. Specifically, defining

$$
\Delta_{e d}^{(i)}=2 \frac{\left(m_{\tilde{d}_{1 i}}+m_{\tilde{d}_{2 i}}\right)-\left(m_{\tilde{e}_{1 i}}+m_{\tilde{e}_{2 i}}\right)}{\left(m_{\tilde{d}_{1 i}}+m_{\tilde{d}_{2 i}}\right)+\left(m_{\tilde{e}_{1 i}}+m_{\tilde{e}_{2 i}}\right)}, \quad i=1,2
$$

where $i$ is the generation index, for the mSUGRA case one finds that $\Delta_{e d}^{(i)}$ are positive and typically a significant fraction. However, for the GNLSP case one has

$$
\left|\Delta_{e d}^{(i)}\right| \ll 1
$$

and often $\left|\Delta_{e d}^{(i)}\right|$ lie in the range much smaller than 1\%. Thus the validity of Eq. (15) implies a high degree of degeneracy of the squark and slepton masses for the first two generations, and the observation of such a degeneracy will provide a strong corroborating evidence along with collider signals for testing the validity of the GNLSP models. Of course, a test of Eq.(15) would require determination of the squark and slepton masses with a certain degree of accuracy.

More generally the lower left hand panel of Fig.(1) exhibits $\Delta_{e d}$ as function of $\Delta_{\text {Co }} \equiv \Delta_{\tilde{g} \widetilde{\chi}^{0}}$ where the gluino NLSP and neutralino LSP coannihilate to produce the consistent relic density observations of WMAP. The analysis of this panel exhibits more generally the results of Table((3) in that one finds that in all these models $\Delta_{e d}$ is relatively small and often negative. We thus arrive at the important general conclusion that in the model where the gluino is the NLSP one gets a compressed sfermion spectrum 8 for the first two generations relative to the squarks, with mass differences between squarks and their slepton counterparts which are typically order a few percent and often less over a wide range of the parameters space. In Fig.(2) the left panel shows that the model GNLSP A $_{\text {can }}$ be discriminated from the bino branch of mSUGRA, while the right panel of Fig.(2) shows that all GNLSP models can be discriminated from mSUGRA. Additionally a comparison of the right and the left panels also allows a discrimination of GNLSP $_{\mathrm{A}}$ from GNLSP $_{\mathrm{B}}$.

\begin{tabular}{||l||l||c|c||c|}
\hline \hline Model & Pattern & $m_{\tilde{e}_{1}}+m_{\tilde{e}_{2}}$ & $m_{\tilde{d}_{1}}+m_{\tilde{d}_{2}}$ & $\Delta_{d e}^{(1)}$ \\
\hline \hline mSUGRA & mSP3 & 5377 & 7652 & $35 \%$ \\
NUSUGRA SU(5) & NUSP13 & 7386 & 7373 & $-0.1 \%$ \\
NUSUGRA SO(10) & NUSP13 & 7369 & 7300 & $-0.1 \%$ \\
\hline \hline
\end{tabular}

Table 3: Exhibition of the mass compression for sleptons and squarks in the first two generations in two typical GNLSP SUGRA models with nonuniversalities and a comparison with a mSP3 model point in the mSUGRA case. Mass splittings between the sleptons and the squarks are seen to be much smaller for the GNSLP models compared to the mSUGRA case.

\footnotetext{
${ }^{8}$ The compressed sfermion spectrum discussed here is very different from the one discussed in 43$]$.
} 


\section{Signature analysis at the LHC}

Gluino Mass and Gluino Production Cross Sections: The production cross sections for gluinos were studied early on [44] and the NLO evaluations have also been given[45]. A particularly interesting situation is the one which is discussed in the preceding sections where the gluino is the NLSP, as this possibility leads to a rather predictive model. Thus one finds that in the GNLSP case the gluino production cross section dominates all other SUSY processes and further the production is controlled to a large degree by a single process which is $g g \rightarrow \tilde{g} \tilde{g}$, i.e., $\sigma_{p p}(\mathrm{SUSY}) \approx \sigma_{p p}(g g \rightarrow \tilde{g} \tilde{g})$ where $\sigma$ (SUSY) is the LHC production cross section including all $2 \rightarrow 2$ SUSY production modes [46]. A numerical analysis of the above is shown in Fig.(3) for the GNLSPs. A consequence of the dominance of the processes $g g \rightarrow \tilde{g} \tilde{g}$ over all others has interesting implications. Specifically it opens up the interesting possibility that a rather precise determination of the gluino mass can be made from a measurement of the production cross section of all SUSY processes. Further since the neutralino is linearly related to the gluino through $\Delta_{\tilde{g} \tilde{\chi}^{0}}$ the LHC data in this case could allow us to determine the neutralino mass with a fair degree of accuracy should the gluino mass be reconstructed.
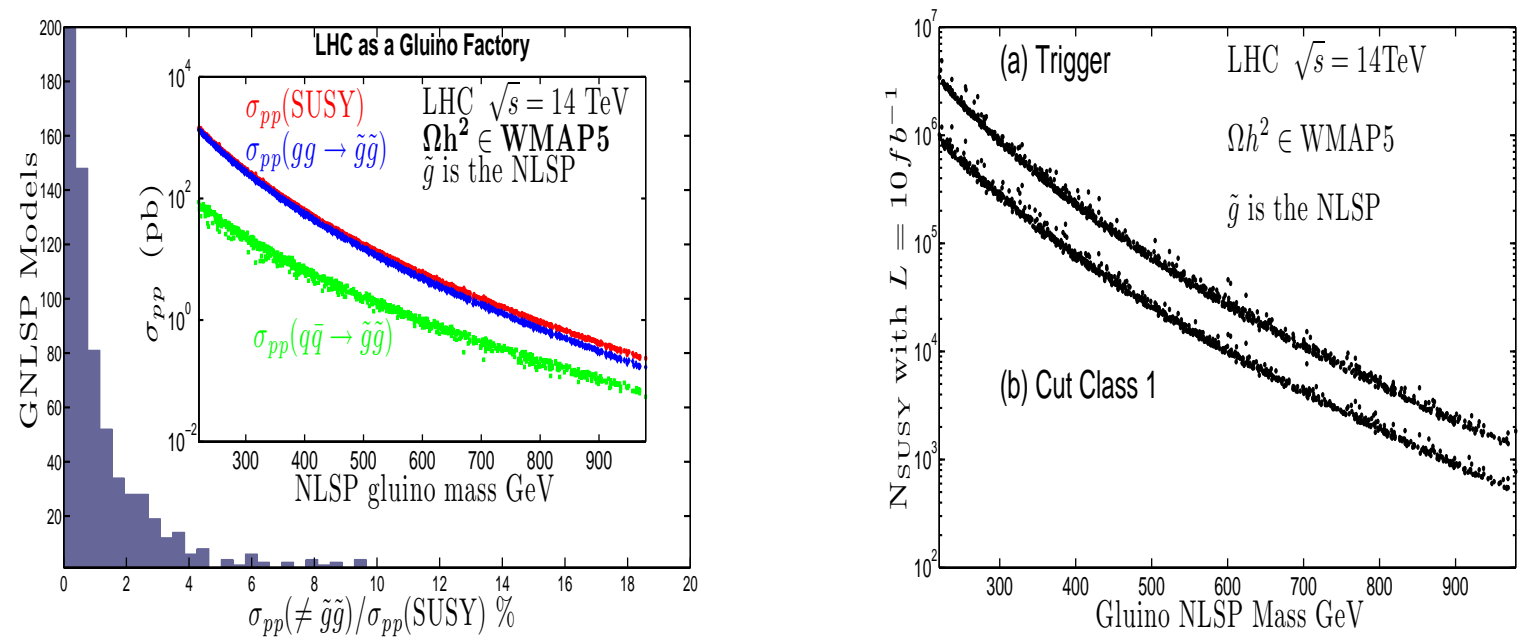

Figure 3: (Color online) Left panel: Display of $p p$ cross sections including the individual production modes arising from subprocesses $g g \rightarrow \tilde{g} \tilde{g}$ and $q \bar{q} \rightarrow \tilde{g} \tilde{g}$ and the total SUSY cross sections plotted as a function of the gluino mass showing the dominance of the $\tilde{g} \tilde{g}$ production process. The analysis of the figures shows that in the GNLSP case the LHC will turn into a gluino factory. Right panel: Total number of SUSY events passing the Level 1 (L1) trigger cuts and post trigger level cuts $\mathrm{C} 1$ as defined in the text. A majority of the events pass the triggers as can be seen by comparing with the left and the right panels of Fig.(3) and taking into account that the right panel of this figure is given for $10 \mathrm{fb}^{-1}$ of luminosity.

It is worthwhile to pause and comment on the sensitivity of the relic density to the codes that 
are often used for its computation. We exhibit this sensitivity in Table(4) where a small dialing of parameters has been done to keep the relic density in the corridor allowed by WMAP. The analysis shows that the sensitivity to the codes is rather small and the GNLSP model is robust in that it appears in all the three codes used in Table(4). In Table(5) we compare the leading order (LO) predictions of PYTHIA and PROSPINO in the GNLSP model for the same parameter point given in Table(4), and show the next to leading order (NLO) prediction using PROSPINO. We also compare the relevant branching ratios. One observes that the squarks decay back into

\begin{tabular}{cccc}
\hline & SuSpect(2.41)[17] & SOFTSUSY(3.0.2)[18] & SPheno(2.2.3)[19] \\
\hline$\widetilde{\chi}^{0}$ & 336.3 & 334.5 & 334.5 \\
$\tilde{g}$ & 382.7 & 379.8 & 381.0 \\
$\tilde{\chi}_{1}^{ \pm}$ & 424.1 & 422.4 & 422.9 \\
$\widetilde{t}_{1}$ & 451.4 & 464.7 & 447.4 \\
$\left(\Omega h^{2}\right)_{\widetilde{\chi}^{0}}$ & 0.115 & 0.105 & 0.117 \\
$\left(\delta_{2}, \delta_{3}\right)$ & $(-0.340,-0.835)$ & $(-0.340,-0.824)$ & $(-0.340,-0.823)$ \\
\hline
\end{tabular}

Table 4: A comparison of the sparticle spectra and of the relic density for a GNLSP model with $\left(m_{0}, m_{1 / 2}, A_{0}, \tan \beta\right)=(1387,792,3026,27)($ all masses in $\mathrm{GeV})$, with $\mu>0$ for $m_{t}=170.9 \mathrm{GeV}$ and $\delta_{2}=-0.340$ with $m_{b}\left(m_{b}\right)$ and $\alpha_{s}\left(M_{Z}\right)$ taken with the default values of MicrOMEGAs (MO). For these models $\widetilde{\chi}_{1}^{ \pm} \lesssim \widetilde{\chi}_{2}^{0}$ (NUSP13). We use MO 2.2CPC for the first two cases and MO 2.07 for SPheno. This particular comparison is made at the perturbative level.

\begin{tabular}{|c|c|c|c|c|}
\hline \multirow{6}{*}{$\begin{array}{c}\text { PYTHIA }[49] \sigma_{\mathrm{LO}} \\
g g \rightarrow \tilde{g} \tilde{g}=70 \mathrm{pb} \\
q \bar{q} \rightarrow \tilde{g} \tilde{g}=6.2 \mathrm{pb} \\
q_{j} g \rightarrow \tilde{q}_{j L} \tilde{g}=1.1 \mathrm{pb} \\
g g \rightarrow \tilde{\tilde{t}}_{1} \tilde{\tilde{t}}_{1}=1.5 \mathrm{pb} \\
\text { else } \ll 1 \mathrm{pb} \\
\sigma_{\mathrm{cICV}}^{\mathrm{LO}}=80 \mathrm{pb}\end{array}$} & & $K_{\mathrm{NLO}}$ & \\
\hline & & $\tilde{g}=84.3 \mathrm{pb}$ & $\frac{\Lambda_{\mathrm{NLO}}}{1.72}$ & $\frac{O_{\mathrm{NLO}}}{145 \mathrm{pb}}$ \\
\hline & & $\tilde{g}=3.12 \mathrm{pb}$ & 1.60 & $5.0 \mathrm{pb}$ \\
\hline & & $\tilde{t}_{1}=0.80 \mathrm{pb}$ & 1.55 & $1.24 \mathrm{pb}$ \\
\hline & & lse $\ll 1 \mathrm{pb}$ & 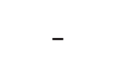 & $20=8$ \\
\hline & & $\mathrm{SY}_{\mathrm{SY}}=88.5 \mathrm{pb}$ & $\Longrightarrow$ & $\sigma_{\mathrm{SUSY}}^{\mathrm{NLO}}=151.6 \mathrm{pb}$ \\
\hline Decay & & BR PYTHIA $[4$ & $\overline{\mathrm{BR}}$ & SUSY-HIT [4] \\
\hline $\begin{array}{r}\tilde{g} \rightarrow\left(b b \widetilde{\chi}^{0}, u \bar{u} \widetilde{\chi}^{0},\right. \\
\tilde{g} \rightarrow \widetilde{\chi}^{0} g \\
\tilde{q}_{L} \rightarrow \tilde{g}(u, d) \\
\widetilde{t}_{1} \rightarrow \widetilde{\chi}^{+} b\end{array}$ & & $\begin{array}{c}(20,61,19) \% \\
- \\
82 \% \\
100 \%\end{array}$ & & $\begin{array}{c}20,61,19) \% \\
0.03 \% \\
86 \% \\
100 \%\end{array}$ \\
\hline
\end{tabular}

Table 5: A specific exhibition of the dominance of the process $g g \rightarrow \tilde{g} \tilde{g}$ in $p p$ collisions at LO and NLO for the GNLSP model point given in Table(4).

a gluino with a large branching ratio. The NLO calculation retains the dominance of the $\sigma_{\tilde{g} \tilde{g}}^{\mathrm{NLO}}$ at the level of $96 \%$, i.e., $\sigma_{\tilde{g} \tilde{g}}^{\mathrm{NLO}}=0.96 \sigma_{\mathrm{SUSY}}^{\mathrm{NLO}}$. We note that the model in Table(5) has a very small branching fraction $\tilde{g} \rightarrow \widetilde{\chi}^{0} g$ (calculated with SUSY-HIT [47] and a very similar suppression is seen with ISAJET [48] $(<0.1 \%)$ ). This is to be contrasted with the GNLSP $_{\text {Co }}$ model (see 

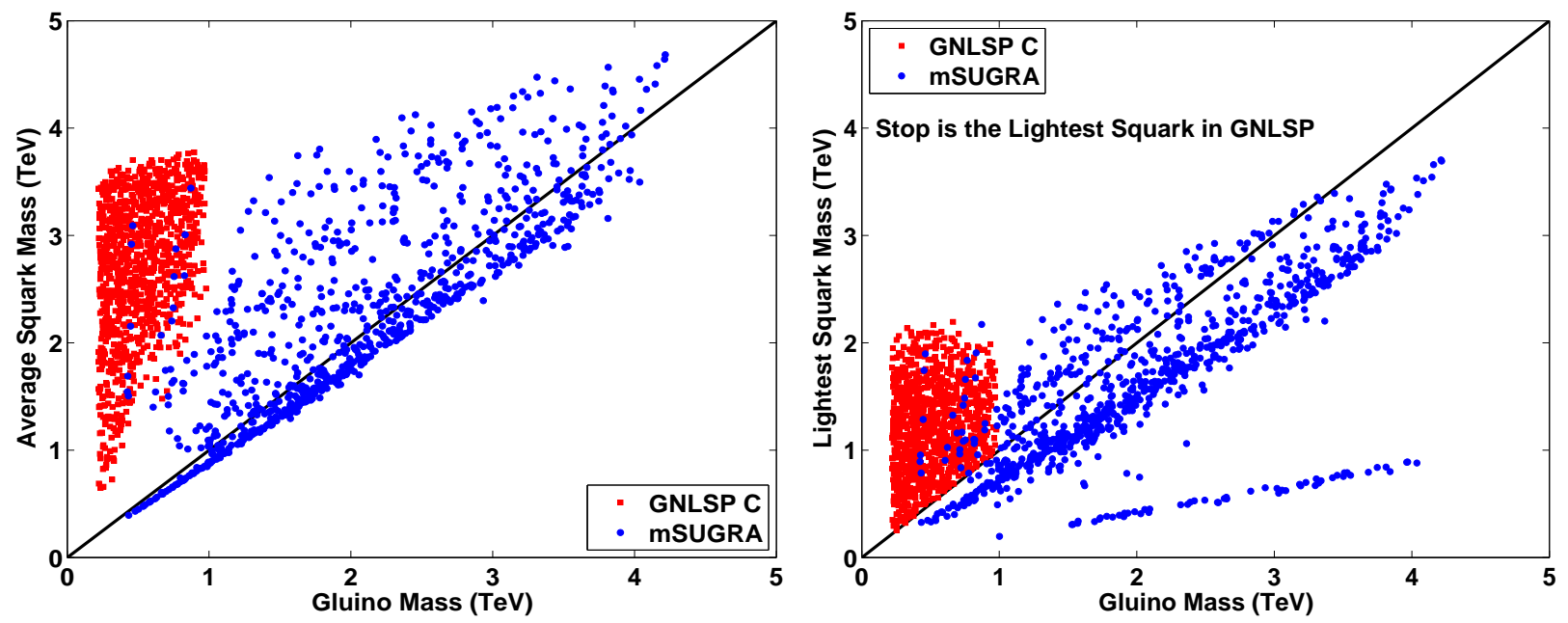

Figure 4: (Color online) Left panel: A display of the average squark mass vs the gluino mass in GNLSP $_{\mathrm{C}}$ and a comparison with mSUGRA. Right panel: A display of the lightest squark mass vs the gluino mass in $\operatorname{GNLSP}_{\mathrm{C}}$ and a comparison with mSUGRA. The gluino mass is relatively light in the GNLSP models with an upper limit of a $\mathrm{TeV}$ under the assumed naturalness assumptions. The lightness of the gluino mass leads to the dominance of the $\tilde{g} \tilde{g}$ over all other sparticle production processes in the GNLSP models.

Eq.(6) ), which has a $\tilde{g} \rightarrow \widetilde{\chi}^{0} g$ of $65 \%$ with SUSY-HIT and $89 \%$ with ISAJET. The gluino decay branching ratios are discussed in further detail later.

In the left panel of Fig.(4) we give a comparison of the average mass of the squarks vs the gluino mass in GNLSP and compare it to that for the mSUGRA model. A similar analysis for the lightest squark mass is given on the right panel. In both cases one finds that aside from a very small region, the spectra from these two models do not overlap. Quite remarkably for the assumed naturalness conditions as described in Sec.(5) the gluino mass in the GNLSP models has an upper limit of about a TeV, while in mSUGRA this limit extends far beyond. On the other hand, the upper limit on the squark masses is quite large extending to several $\mathrm{TeV}$ in both cases. It is the relative lightness of the gluino mass in the GNLSP case that enhances the production of the gluinos relative to all other SUSY production processes such as $\tilde{g} \tilde{q}$ and $\tilde{t}_{1} \overline{\tilde{t}}_{1}$ at the LHC. This further explains the result of Fig.(3) and of Table(15) which show that the $\tilde{g} \tilde{g}$ production cross section dominates over all others. Thus in effect, in the GNLSP case, the LHC will become a gluino factory. Nonetheless, the production cross section for the squarks is still significant and their production could be detectable with efficient cuts. For example, the stop is usually the lightest squark in GNLSP models, so its production could be significant, and it can decay via $\tilde{t}_{1} \rightarrow \tilde{\chi}^{+}+b \rightarrow W^{+}+b+\tilde{\chi}^{0}$. Since the $W^{+}$has decays into $l^{+}+\nu$, the stop will have some leptonic signatures while the gluino is a pure jet signal. An analysis of these leptonic signals requires a further dedicated analysis which is left for a future analysis. We also note that superficially the overlap of the few GNLSP points with the mSUGRA point might be 
construed as the existence of a degeneracy for these parameter points. However, we need to keep in mind that what is plotted is an average squark mass. Further, as already discussed there is a significant splitting between the squark masses and the slepton masses for the mSUGRA model for the first two generations while there is very little splitting in this case for the GNLSP model. Thus at the very least the slepton-squark splittings lift any perceived degeneracy indicated in Fig.(4). We should also point out that although the production cross section of squarks and gluinos can be comparable for GNLSP models and for mSUGRA models, their LHC signatures tend to be significantly different due to the mass spectra of sparticles being rather different and specifically this is so since the gluino is the NLSP in the class of models we consider.

Early LHC Discovery Prospects at Low Luminosity: Each GNLSP model is subject to the experimental constraints as discussed in Section(3). We investigate the LHC signatures of 1070 such GNLSP models with PYTHIA coupled to PGS4 [52]. Branching fractions are computed with SUSY-HIT and fed directly into the PYTHIA decay table via the SUSY Les Houches Accord (SLHA) [51] interface. For the GNLSP models, this is quite important as one must take into account the radiative decay $\tilde{g} \rightarrow \widetilde{\chi}^{0} g$ which can be substantial for this class of models. The LHC detector simulation proceeds with PGS4 with the Level 1 (L1) triggers designed to mimic the Compact Muon Solenoid detector (CMS) specifications [53] with the LHC detector card. Specifically the L1 trigger level cuts that are imposed are as follows [52]: (1) inclusive isolated lepton $(\mu / e)(30 \mathrm{GeV})$; (2) lepton plus jet (20 GeV, $100 \mathrm{GeV})$; (3) isolated di-leptons (15 GeV); (4) di-leptons plus jet $(10 \mathrm{GeV}, 100 \mathrm{GeV})$; (5) isolated di-leptons (10 GeV); (6) isolated lepton plus isolated $\tau(15 \mathrm{GeV}, 45 \mathrm{GeV})$; (7) isolated di-tau $(60 \mathrm{GeV}) ;(8)$ inclusive isolated photon $(80 \mathrm{GeV})$; (9) isolated di-photon $(25 \mathrm{GeV})$; (10) inclusive $\not P_{T}(90 \mathrm{GeV}) ;(11)$ inclusive single-jet $(400 \mathrm{GeV}) ;(12)$ jet plus $\not P_{T}(180 \mathrm{GeV}, 80 \mathrm{GeV}) ;(13)$ acoplanar jet and $\not P_{T}$ with $(1<\Delta \phi<2)$ $(100 \mathrm{GeV}, 80 \mathrm{GeV})$ (jet, $\left.\not P_{T}\right) ;(14)$ acoplanar di-jets with $(\Delta \phi<2)(200 \mathrm{GeV})$. Muon isolation is controlled by employing the cleaning script in PGS4. SM backgrounds have been generated with QCD multi-jet production due to light quark flavors, heavy flavor jets $(b \bar{b}, t \bar{t})$, Drell-Yan, single $Z / W$ production in association with quarks and gluons $(Z / W+$ jets $)$, and $Z Z, W Z, W W$ pair production. The standard criteria for the discovery limit are imposed, namely, that the SUSY signal is taken as discoverable if the number of SUSY events exceeds $5 \sqrt{N_{\mathrm{SM}}}$ or 10 whichever is larger, i.e., $\mathrm{N}_{\mathrm{SUSY}}>\operatorname{Max}\left\{5 \sqrt{N_{\mathrm{SM}}}, 10\right\}$. We implement several classes of post trigger level cuts to analyze the SM background and our event samples.

Post Trigger Level Cuts: As already noted the dominant sparticle production process at the LHC will be $g g \rightarrow \tilde{g} \tilde{g}$, and the dominant decays of the gluino are $q \bar{q} \widetilde{\chi}^{0}$ and depending on the particular part of the parameter space the decay $\tilde{g} \rightarrow g \tilde{\chi}^{0}$ [54, 55, 9] can also be large, and in fact can dominate over the 3 body decay, depending on a confluence of the following: (a) the lightness of the gluino, (b) the largeness of the squark masses, and (c) the neutralino mixing matrix. While a heavy gluino can decay into quarks and squarks and into weak gauge bosons $W^{ \pm}, Z[56$, these decays are either kinematically forbidden, such as into quarks and squarks, or are highly suppressed for the GNLSP models we are discussing here. The above implies that GNLSP models will lead to a preponderance of jet signatures. In our analysis we impose three different sets of post trigger level cuts to optimize the signal and reduce the background from the 
standard model processes. We classify these as: (i) Class 1 (C1), (ii) Class 2 (C2), and (iii) Class 3 (C3) post trigger level cuts. We discuss these in some detail below and discuss the signals that are best detected with these three classes of cuts. Before proceeding further, we note that the missing transverse momentum is an important cut both as a trigger level as well as a post trigger level cut allowing one to increase the signal relative to the background. Thus SUSY models with a LSP which is massive tend to produce events at the hadron colliders with a larger missing energy. In order to suppress the Standard Model background, usually a large missing transverse momentum cut is employed. We note further that since $b$ and $\bar{b}$ are produced in the processes $g g \rightarrow q \bar{q}, q \tilde{g}, \tilde{g} \tilde{g}$ as well as with a certain fraction in $g g \rightarrow \tilde{g} \tilde{g}$ with subsequent decays of $\tilde{g}$ (this latter case being the most relevant one discussed here), one has a significant number of b jets produced in these events. Thus b-tagging is a useful instrument in their identification. These features will be seen in the post trigger level cuts we discuss below.

\section{(i) Class 1 Post Trigger Level Cuts (C1)}

1. Electrons, and muons with $P_{T}>10 \mathrm{GeV}$ (where $P_{T}$ is the transverse momentum) and $|\eta|<2.4$ (where $\eta$ is pseudorapidity) are selected.

2. Jets with $P_{T}>60 \mathrm{GeV}$ and $|\eta|<3$ are selected.

3. Events with $\not P_{T}>200 \mathrm{GeV}$ are selected.

Since the GNLSP models have a gluino which lies close to the LSP consistent with the relic density constraints, a large portion of events generated by the SUSY processes have a less energetic jet signal and a relatively smaller missing energy than may be observed, for example, in the case of stau coannihilation (see [35] for analysis of such a signature). Taking the above into account we modified the imposed C1 post trigger level cuts in $\mathrm{C} 2$ and $\mathrm{C} 3$ which are to be discussed below. As already emphasized, the SUSY production cross section are dominated by gluino pair production and decays of the gluino. Therefore, we only select events that have at least two jets that pass our jet selection condition. We also investigate the effects of putting a softer missing $P_{T}$ cut and jet $P_{T}$ cut.

(ii) Class 2 Post Trigger Level Cuts (C2)

1. Electrons, and muons with $P_{T}>10 \mathrm{GeV}$ and $|\eta|<2.4$ are selected.

2. Jets with $P_{T}>50 \mathrm{GeV}$ and $|\eta|<3$ are selected.

3. Events with $\not P_{T}>150 \mathrm{GeV}$ are selected.

4. Events with at least 2 jets are selected.

The dominant SM background for GNLSP models are from QCD, $Z / W+$ jets, $b \bar{b}$, and $t \bar{t}$ and we focus on these in Fig.(5). In the left panel of Fig.(15) we give an analysis of these backgrounds at the LHC with events/bin $/ \mathrm{fb}^{-1}$ as a function of the azimuthal angle $\Delta \phi\left(\right.$ jet $_{1}$, jet $\left.{ }_{2}\right)$ between the 

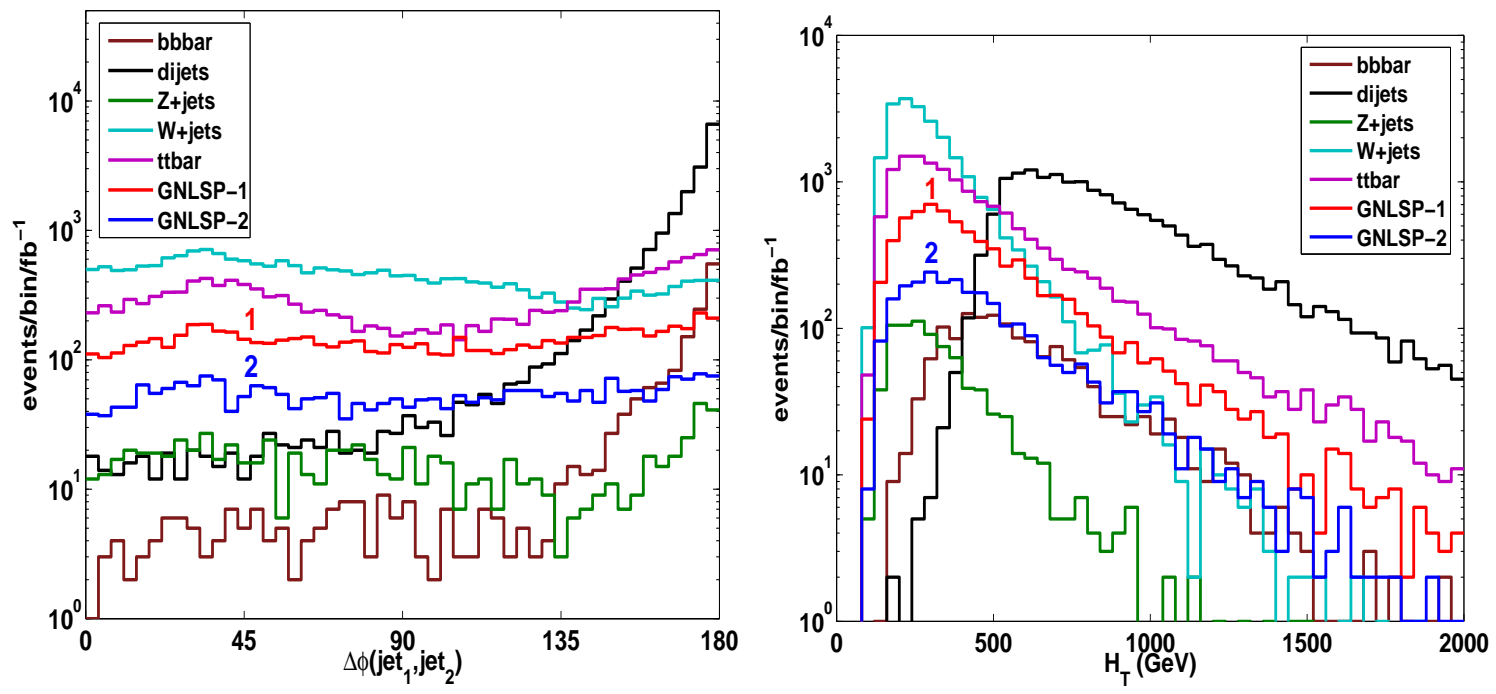

Figure 5: (Color online) Left panel: An analysis of events/bin $/ \mathrm{fb}^{-1}$ as a function of the azimuthal angle $\Delta \phi\left(\right.$ jet $_{1}$ jet $\left._{2}\right)$ between the two hardest jets in the GNLSP model relative to the SM backgrounds with C2 cuts. Right panel: $H_{T}=\sum_{\text {jets }} P_{T}$ distributions of GNLSP models and SM backgrounds with $\mathrm{C} 2$ cuts. These distributions act as a guide for implementing the C3 cuts as discussed in the text.

two leading jets. We also show the distributions for two GNLSP model points with masses of 400 and $500 \mathrm{GeV}$ respectively. A similar analysis is presented but as a function of the total jet $P_{T}$ (labeled $H_{T}$ ) in the right panel of Fig.(5), where again we also exhibit the distributions for two GNLSP model points. The analysis provides a good starting point for charting out the post trigger level cuts needed to reduce the background and enhance the signal. Thus the analysis suggests that one may cut out the events that have a large $\Delta \phi\left(\right.$ jet $_{1}$, jet $\left.{ }_{2}\right)$ as this cut will suppress the QCD background due to light quark flavors, $b \bar{b}$ and $t \bar{t}$. A veto on isolated electrons or muons was applied to reject the background events containing $W$ or $Z$ leptonic decays. A simple counting of events after applying C2 cuts reveals that the GNLSP models are nearly lepton free, as is the case for the $b \bar{b}$ and di-jets background. However, the standard model backgrounds due to Drell-Yan process and $(Z / W+$ jets $)$ have $(31 \% \sim 36 \%)$ of the total events that contain electrons or muons, while for $t \bar{t}$ background it is about $45 \%$. The $Z Z, W Z, W W$ pair production resulting in multi-leptonic backgrounds have an even larger percentage of leptonic events. Therefore, the $e / \mu$ veto significantly enhances the GNLSP signals over the standard model background. This leads us to investigate cut class C3. 

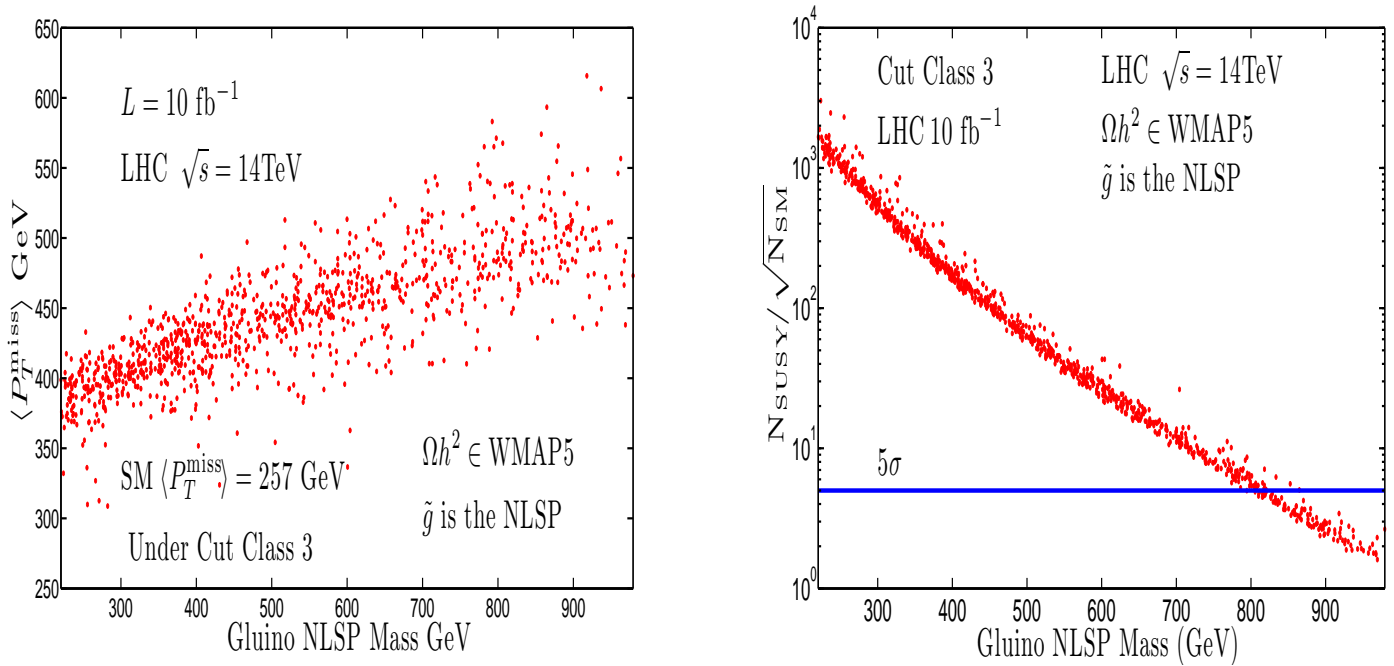

Figure 6: (Color online) The analysis above is with post trigger level cuts C3 and with an integrated luminosity of $10 \mathrm{fb}^{-1}$. Left panel: average $P_{T}$ vs the gluino mass; Right panel: the discovery reach in SUSY events vs the gluino mass.

(iii) Class 3 Post Trigger Level Cuts (C3)

1. Apply cut set $\mathrm{C} 2$.

2. Electron or muon veto is imposed.

3. $H_{T} \equiv \sum_{\text {jets }} P_{T}>400 \mathrm{GeV}$.

4. The azimuthal angle $\Delta \phi\left(\right.$ jet $_{1}$, jet $\left._{2}\right)$ between jet1 (the hardest jet) and jet2 (the second hardest jet $)$ is chosen so that $\Delta \phi\left(\right.$ jet $_{1}$, jet $\left._{2}\right)<3 \pi / 4$.

5. The azimuthal angle $\Delta \phi\left(\right.$ jet $\left._{1}, \not_{T}\right)$ between jet1 (the hardest jet) and $\not P_{T}$ is chosen so that $\Delta \phi\left(\right.$ jet $\left._{1}, \not_{T}\right)>\pi / 2$.

6. The azimuthal angle $\Delta \phi\left(\right.$ jet $\left._{2}, \not_{T}\right)$ between jet2 (the second hardest jet) and $\not P_{T}$ is chosen so that $\Delta \phi\left(\right.$ jet $\left._{2}, P_{T}\right)>\pi / 4$.

As is indicated from the preceding discussion, jets with and without tagged b jets are important signals for the discovery of the GNLSP models. Another important signature is $\left\langle P_{T}\right\rangle$, which is the average magnitude of the missing transverse momentum, where the average extends over all events passing the cuts. We discuss now several signatures for the GNLSP models as given in Fig.(6). The analysis of Fig.(6) is given at $\sqrt{s}=14 \mathrm{TeV}$ under post trigger level cuts C3 with an integrated luminosity of $10 \mathrm{fb}^{-1}$. The left panel of Fig.(6) exhibits the average $\not_{T}$ as a function of the gluino mass. Here one finds that essentially all of the parameter points of the GNLSP 

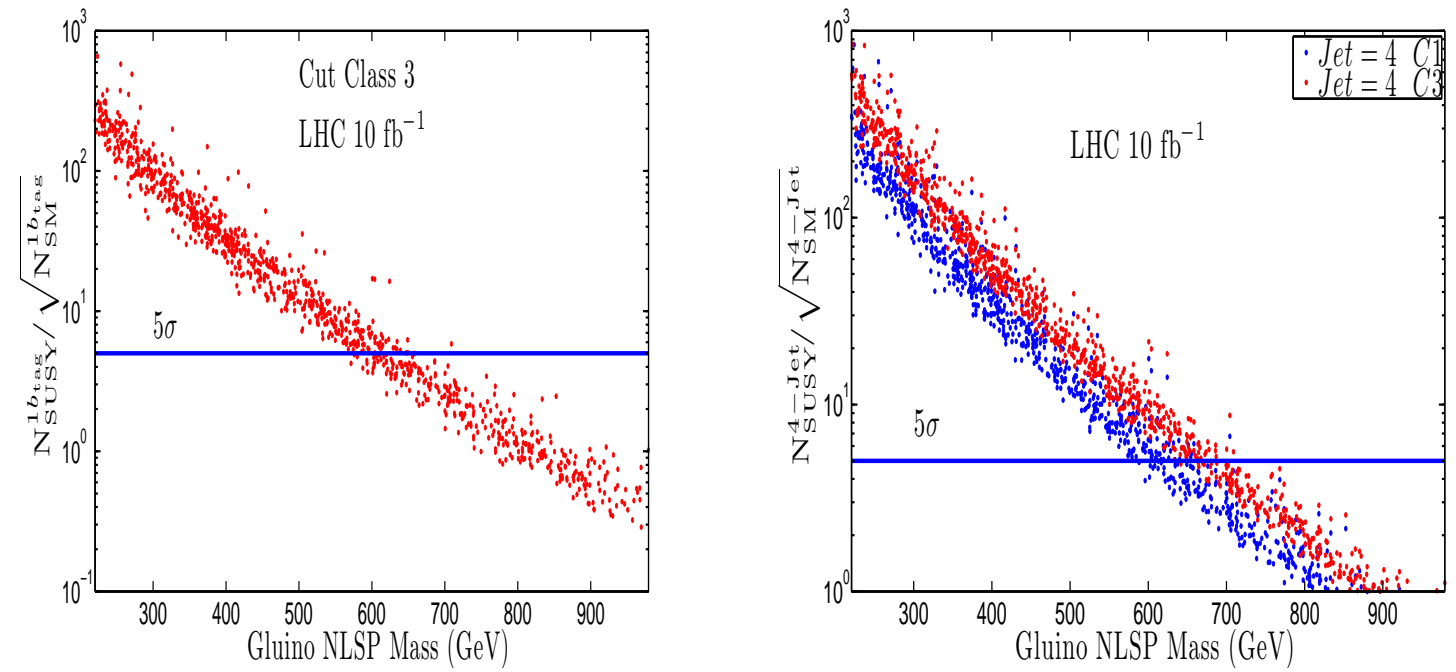

Figure 7: (Color online) All Cuts and constraints as in Fig.(6): Left panel: the discovery reach with 10/fb for SUSY events with 1 tagged b-jet vs the gluino mass, and Right panel: A comparison of post trigger level cuts $\mathrm{C} 1$ and $\mathrm{C} 3$ for the discovery of a 4-jet signal with an integrated luminosity of $10 \mathrm{fb}^{-1}$ at the LHC. It is seen that the specialized cuts C3 enhance the 4J reach by roughly $100 \mathrm{GeV}$ relative to the post trigger level cuts $\mathrm{C} 1$. The C3 cuts reduce the SM background while allowing a large n-jet signal for $n \geq 2$ with the largest signal to background enhancement appearing for $n=4$.

models have an average $\not_{T}$ which is larger, and often significantly larger, than for the standard model case for which the average $P_{T}$ is found to be $\sim 257 \mathrm{GeV}$ under the same set of cuts. The right panel illustrates the ratio $N_{\mathrm{SUSY}} / \sqrt{N_{\mathrm{SM}}}$ vs the gluino mass where $N_{\mathrm{SUSY}}$ is the total number of SUSY events and $N_{\mathrm{SM}}$ is the standard model background, again with the imposition of post trigger level cuts C3. Here one finds that the ratio $N_{\mathrm{SUSY}} / \sqrt{N_{\mathrm{SM}}}$ lies above $5 \sigma$ discovery limits for gluino masses up to $800 \mathrm{GeV}$ making this ratio an important channel for the discovery of the GNLSP models.

The left panel of Fig.(17) demonstrates that the events containing a single tagged b-jet leads to a discoverable signal over a wide range of gluino masses. Specifically this panel gives the ratio $N_{\mathrm{SUSY}}^{1 b_{j e t}} / \sqrt{N_{\mathrm{SM}}^{1 b_{j e t}}}$ vs the gluino mass where $N_{\mathrm{SUSY}}^{1 b_{j e t}}$ is the number of SUSY events with 1 tagged b-jet and $N_{\mathrm{SM}}^{1 b_{j e t}}$ is the number of standard model events with 1 tagged b-jet under the imposition of class C3 cuts. This channel provides a $5 \sigma$ discovery for gluino masses up to about $600 \mathrm{GeV}$.

We briefly comment on the ability to tag b-jets in this case. Naively one would expect that for cases in which the dominant decays are $\tilde{g} \rightarrow \widetilde{\chi}^{0} q \bar{q}$, with sizeable branchings in b quarks, that the b-jet events would be a gold plated signal. However, here the b's come out rather soft since the mass splitting of the $\left(\tilde{g}-\widetilde{\chi}^{0}\right)$ is rather small, typically around $50 \mathrm{GeV}$ (the phenomenon 
responsible for the satisfaction of the relic density is precisely this mass split). While the ability to tag the b's is possible, it is indeed more difficult than the canonical situation seen on the Hyperbolic Branch of REWSB [40] (for recent work on b-tagging analyses see [42, 57, 37]).

Nonetheless, as discussed above the signal for singly tagged b-jets is strong enough that it can be a useful one. The right panel of Fig.(17) we compare the 4-jet discovery limits under $\mathrm{C} 1$ and $\mathrm{C} 3$ cuts. Here it is found that the SM background drops by an appreciable amount as one goes from $\mathrm{C} 1$ cuts to $\mathrm{C} 3$ cuts, allowing one to extend the discovery limit in 4-jet channel by over $100 \mathrm{GeV}$ with $\mathrm{C} 3$ cuts relative to imposing the $\mathrm{C} 1$ cuts in this channel. Amongst the classes of n-jet events, we find this channel to be the most enhanced when passing from $\mathrm{C} 1$ to C3. Combining all the channels analyzed in Fig.(6) one finds that gluino masses up to $800 \mathrm{GeV}$ are discoverable by this technique for a GNLSP model with just $10 \mathrm{fb}^{-1}$ of integrated luminosity. Thus the validity of the GNLSP models can be tested with first data from the LHC.

\section{Dark matter detection in the GNLSP models}

Many of the GNLSP model points have an LSP neutralino which is bino like. However, there is also a significant set of models that have an LSP with large higgsino components (many of which are at high $\tan \beta$ but this is not exclusively so). The large $\tan \beta$ parameter points are easily spotted by examining the $\tan \beta$ vs $A_{0} / m_{0}$ plot in Fig.(1). The GNLSP models have important implications for the direct detection of neutralino dark matter. An analysis of the spin independent and spin dependent cross sections in dark matter experiments is given in Fig. (8) implemented with MicrOMEGAs. Included are published limits from the ZEPLIN-III experiment [58], the first five-tower CDMS data[59] and the XENON 10 results [60]. Projected limits (indicated by ${ }^{*}$ ) from CDMS and LUX are also shown [61]. The $\sigma_{S I}$ vs $m_{\tilde{\chi}^{0}}$ analysis shows some interesting results. First one finds that there are a class of GNLSP models which are beginning to be constrained by the direct detection dark matter experiments. These models would also produce large $\tilde{g} \tilde{g}$ production cross sections and will be easily visible at the LHC. However, interestingly there is another class of low mass neutralino (and low mass gluino) models which have rather small spin independent cross sections which are outside the reach of the direct detection experiments in the foreseeable future. Nonetheless these models would still lead to rather large $\tilde{g} \tilde{g}$ production cross sections and hence will be visible at the LHC. Thus the LHC can detect many of the GNLSP models which are most likely inaccessible to the direct detection dark matter experiments. On the flip side, for the large set of low mass GNLSP models one still has the possibility of a very light neutralino (gluino) with a sizeable higgsino component and consequently a large spin independent cross section. Thus if a light gluino is indeed indicated early on at the LHC, it may also provide a hint of the size of the dark matter signal in direct detection of dark matter. We note in passing that a plot of $\sigma_{S I}$ vs $m_{\tilde{g}}$ looks very similar to the left panel of Fig.(8) as the gluino and neutralino mass are related at the low scale via $m_{\tilde{g}}=\left(1+\Delta_{\tilde{g} \tilde{\chi}^{0}}\right) m_{\tilde{\chi}^{0}}$. 

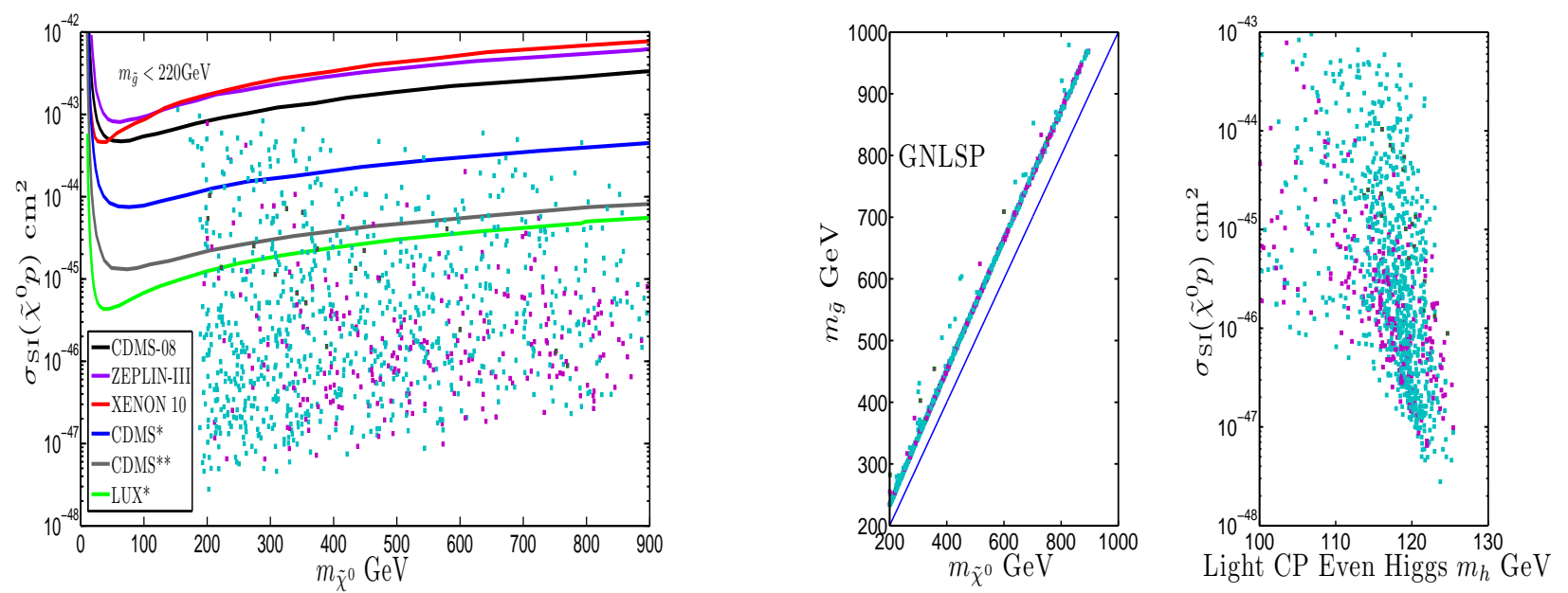

Figure 8: (Color online) Left panel: An exhibition of the spin independent cross section $\sigma_{S I}$ as a function of the neutralino mass. It is seen that there are a large number of models corresponding to $\sigma_{S I}$ in the range $10^{-46} \mathrm{~cm}^{2}$ and below, which would be inaccessible in direct dark matter searches in the foreseeable future. However, many of these models especially those with low values of $m_{\tilde{\chi}^{0}}$ (and hence of $m_{\tilde{g}}$ ) would be discoverable at the LHC even with low luminosity. Right panel (1): The explicit scaling relation between $m_{\widetilde{\chi}^{0}}$ and $m_{\tilde{g}}$ for the GNLSP models (also shown purely for visual reference is a line representing $m_{\tilde{\chi}^{0}}=m_{\tilde{g}}$ ). Right panel (2): An analysis of $\sigma_{S I}$ vs the light Higgs boson mass illustrating that a large portion of NUSP13 (light blue) has a Higgs boson near $120 \mathrm{GeV}$ while NUSP14 is given in dark (magenta).

We note that in the GNLSP class of models, the direct annihilations of $\widetilde{\chi}^{0} \widetilde{\chi}^{0}$ into electron positron pairs is helicity suppressed. For example, we obtain $\langle\sigma v\rangle_{\widetilde{\chi}^{0} \widetilde{\chi}^{0} \rightarrow e^{+} e^{-}} \sim 5 \times 10^{-30} \mathrm{~cm}^{3} / \mathrm{s}$ (at $v / c=.002$ ) for the model of Table(5) while the self annihilation into $W^{+} W^{-}$are equally small. Annihilations into $\tau \bar{\tau}$ are found to be the largest (this particular model has $\langle\sigma v\rangle_{\widetilde{\chi}^{0} \widetilde{\chi}^{0} \rightarrow \tau \bar{\tau}} \sim$ $\left.2 \times 10^{-27} \mathrm{~cm}^{3} / \mathrm{s}\right)$. Thus a significant boost will be needed to explain the recent cosmic ray excess 62 .

\section{GNLSP Benchmarks}

It is useful to give benchmarks for the three GNLSP models A, B, and C discussed in Sec.(2). As mentioned above, we go beyond the perturbative calculation, and include the Sommerfeld enhancement of the cross section in these benchmarks. In Table([6] we give benchmarks for model GNLSP $_{\text {A }}$. The benchmarks are chosen to exhibit a significant diversity in the input values. Some of the low lying spectrum as well as the spin independent cross section $\sigma_{S I}\left(\tilde{\chi}^{0} p\right)$ and the spin dependent cross section $\sigma_{S D}\left(\tilde{\chi}^{0} p\right)$ corresponding to Table([6]) are exhibited in Table(7). The analysis of Table(7) shows a variation over two orders of magnitude for $\sigma_{S I}\left(\tilde{\chi}^{0} p\right)$. As 
discussed already the variation arises due to changes in the higgsino vs the bino component of the LSP. Similar benchmarks for model GNLSP $_{B}$ are given in Table(8) and the corresponding light sparticle spectrum and the corresponding spin independent cross section $\sigma_{S I}\left(\tilde{\chi}^{0} p\right)$ and the spin dependent cross section $\sigma_{S D}\left(\tilde{\chi}^{0} p\right)$ are exhibited in Table(9). Finally, a similar analysis for the model GNLSP ${ }_{\mathrm{C}}$ is given in Table(10) and Table(11).

\section{Conclusions}

In the above we have given an analysis of a class of models with nonuniversalities which lead to a gluino as the NLSP. Several important observations emerge from this analysis which have bearing on the observation of sparticles at the LHC. Perhaps the most important of these is that if the gluino is the NLSP, then the $g g \rightarrow \tilde{g} \tilde{g}$ cross section at the LHC dominates over all others in the GNLSP models. The dominance of the gluino production and the fact that the $\tilde{g} \tilde{g}$ production cross sections are large implies that the observation of supersymmetry via the gluino production can occur with the first data from the LHC. It is found that the dominant signal of the gluino NLSP model are multi-jets, tagged b-jets and missing energy and it is possible to devise post trigger level cuts which discriminate these models above the standard model backgrounds. Such cuts which reduce the background and enhance the signal to the background ratio were devised and implemented in this paper. We note also that the inverse of the LHC process, namely $\tilde{g} \tilde{g} \rightarrow g g$, is largely responsible for the satisfaction of the relic density when the neutralino and the gluino coannihilate. Further, the analysis of GNLSP models reveals that there exists a significant region of the parameter space in these models where the neutralino has a large higgsino content, and the neutralino-proton spin independent scattering cross section is sizeable and can be probed with the current experimental sensitivity, and sensitivities that would be achievable in future experiments. However, there are also other regions of the parameter space where the neutralino is mostly bino like and in this case the spin independent cross sections can fall well below the current experimental sensitivity, and well below the sensitivity that would be achievable in the near future experiments. Interestingly, the bino cases, though difficult to discover in dark matter experiments, can be accessible at the LHC since the gluino mass in these models lies within the reach of the LHC even at low luminosities. Another aspect of the GNLSP models was also discussed which relates to the compressed nature of the sfermion mass spectrum relative to the case of universal gaugino masses. Here the sleptons in the first two generations could be almost degenerate in mass and often even heavier than their squark counterparts. Several benchmarks for the GNLSP models were also given to facilitate further work. It was also pointed out that a test of the GNLSP models can be done with just $10 \mathrm{fb}^{-1}$ of integrated luminosity and thus it is one of the models that can be checked with the early data at the LHC. Finally, we emphasize once again that if the gluino is an NLSP then the production of gluinos will dominate all other sparticle production making the LHC effectively a gluino factory.

Acknowledgments: This research is supported in part by NSF grants PHY-0653342 (Stony Brook) and PHY-0757959 (Northeastern). The work of DF was also supported in part by Office of the Vice-Provost at Northeastern University. 


\section{Appendix A: Gaugino mass sum rules for GUTS with nonuni- versalities}

Each of the mass ratios listed in Table(2) gives two gaugino mass relations at the GUT scale. Of these one is 'unstable' to the inclusion of a singlet $F$ term breaking while the other one is 'stable' and remains valid when one includes a singlet $F$ term breaking along with the nonsinglet breaking. Below we list only the 'stable' mass relations. The mass relations are labeled numerically (1)-(23) as in Table(2). They are9

$$
\begin{array}{rll}
(1)+(7)+(10):-5 M_{1}+3 M_{2}+2 M_{3}=0, & (2): & M_{1}+3 M_{2}-4 M_{3}=0, \\
(3):-M_{1}+9 M_{2}-8 M_{3}=0, & (4): & 5 M_{1}+3 M_{2}-8 M_{3}=0, \\
(5):-5 M_{1}+9 M_{2}-4 M_{3}=0, & (9): & 5 M_{1}+M_{2}-6 M_{3}=0, \\
(13): M_{1}+M_{2}-2 M_{3}=0, & (14): & -25 M_{1}+9 M_{2}+16 M_{3}=0, \\
(16):-5 M_{1}+M_{2}+4 M_{3}=0, & (18): & 5 M_{1}+11 M_{2}-16 M_{3}=0, \\
(20): 5 M_{1}+7 M_{2}-12 M_{3}=0, & (23): & -5 M_{1}+9 M_{2}-4 M_{3}=0 .
\end{array}
$$

In addition to the above, models (6), (8), (11), (12), (15), (17), (22) in Table(2) satisfy the relation $M_{2}=M_{3}$, while model (19) in Table(2) satisfies the relation $M_{1}=M_{3}$, and model (21) in Table(2) satisfies the relation $M_{1}=M_{2}$. These mass relations would be appropriately modified at low scales by the renormalization group evolution. Thus at the one loop level the mass relations at the electroweak scale are

$$
(r-1) \frac{\alpha_{1}(0)}{\alpha_{1}(Q)} M_{1}(Q)+\frac{\alpha_{2}(0)}{\alpha_{2}(Q)} M_{2}(Q)-r \frac{\alpha_{3}(0)}{\alpha_{3}(Q)} M_{3}(Q)=0,
$$

where $r$ is the ratio as given by Table(2). Assuming one can determine with accuracy the gaugino masses, these mass relations can be a useful indicator of the specific $F$ type breaking and hence of the type of nonuniversal SUGRA model one has at the GUT scale.

\section{Appendix B: An analytic analysis of quasi-degeneracy of LSP and GNLSP and of the sfermion mass compression}

Here we give a one loop analysis of how the quasi degeneracy of the LSP and of the GNLSP comes about and then discuss a similar phenomenon for the squarks and the sleptons for the first two generations. We begin with the gaugino masses which at the GUT scale obey the nonuniversality condition of Eq.(1) which we restate in more compact notation as follows: For model $i$ we have

$$
M_{1}^{(i)}=\gamma_{1}^{(i)} m_{1 / 2}, \quad M_{2}^{(i)}=\gamma_{2}^{(i)} m_{1 / 2}, M_{3}^{(i)}=\gamma_{3}^{(i)} m_{1 / 2}
$$

\footnotetext{
${ }^{9}$ The stability of the gaugino mass relations to the inclusion of singlet breaking is easily seen by noting that the sum of the co-efficients of $M_{1}, M_{2}$ and $M_{3}$ in each of the mass relations in Eq. (16) vanishes.
} 
where

$$
\gamma_{1}^{(i)}=\left(1+a_{i} \alpha_{i}\right), \gamma_{2}^{(i)}=\left(1+b_{i} \alpha_{i}\right), \gamma_{3}^{(i)}=\left(1+c_{i} \alpha_{i}\right)
$$

With the above boundary conditions, the gaugino masses at the electroweak scale $t=\ln \left(M_{G}^{2} / Q^{2}\right)$, where $M_{G}$ is the GUT scale and $Q$ is the electroweak scale, are given by

$$
M_{a}^{(i)}(t)=\gamma_{a}^{(i)} \alpha_{a}(t) m_{1 / 2}, \quad a=1,2,3
$$

Here a $=1,2,3$ correspond to the $U(1), S U(2)$ and $S U(3)$ gauge groups, and $\alpha_{a}(t)$ are the corresponding fine structure constants for these groups at the electroweak scale. For the mSUGRA case, $\gamma_{a}^{(i)}=1$, and one simply has that at one loop

$$
M_{1}(t): M_{2}(t): M_{3}(t)=\alpha_{1}(t): \alpha_{2}(t): \alpha_{3}(t) .
$$

Using the experimental values of the gauge coupling constants at the electroweak scale one finds the three gaugino masses roughly in the ratio $1: \sim 2: 5-6$. In this case the gaugino masses are split in a very significant way. However, in the presence of nonuniversalities the ratios will be modified in a very different way. Thus with the inclusion of the modifications $\gamma_{a}$, the three gaugino masses at the electroweak scale will be roughly in the ratio $\gamma_{1}: \sim 2 \gamma_{2}: \sim(5-6) \gamma_{3}$. It is clear then that the choice $\gamma_{3} / \gamma_{1} \sim(1 / 5-1 / 6)$ will make masses of gaugino 1 and of gaugino 3 almost degenerate. Of course, more realistically there would be mixings between the gauginos and the higgsinos and the mass eigenstates will be admixtures of these. Thus the mass relation of the lightest neutralino and of the gluino will be more complicated. Still the above approximation may roughly hold when the neutralino is mostly a Bino.

Nonuniversalities also enter in the masses for the squarks and for the sleptons. For the first two generation down squarks one finds

$$
\begin{array}{r}
m_{\tilde{d}_{i L}}^{2}(t)=m_{0}^{2}+m_{d i}^{2}+\tilde{\alpha}_{G}\left[\frac{8}{3} \tilde{f}_{3}+\frac{3}{2} \tilde{f}_{2}+\frac{1}{30} \tilde{f}_{1}\right] m_{1 / 2}^{2}+\left(-\frac{1}{2}+\frac{1}{3} \sin ^{2} \theta_{W}\right) M_{Z}^{2} \cos (2 \beta), \\
m_{\tilde{d}_{i R}}^{2}(t)=m_{0}^{2}+m_{d i}^{2}+\tilde{\alpha}_{G}\left[\frac{8}{3} \tilde{f}_{3}+\frac{8}{15} \tilde{f}_{1}\right] m_{1 / 2}^{2}-\frac{1}{3} \sin ^{2} \theta_{W} M_{Z}^{2} \cos (2 \beta),
\end{array}
$$

where

$$
\tilde{f}_{a}=\gamma_{a}^{2} f_{a}, \quad f_{a}(t)=\frac{1}{\beta_{a}}\left(1-\frac{1}{\left(1+\beta_{a} t\right)^{2}}\right),
$$

where $\beta_{a}=b_{a} \tilde{\alpha}_{a}(0), \tilde{\alpha}_{a}(0)=\alpha_{a} / 4 \pi$, and $b_{a}=(33 / 5,1,-3)$ for the gauge groups $U(1), S U(2)$ and $S U(3)$. For the case of the first two generations of charged leptons one has

$$
\begin{array}{r}
m_{\tilde{e}_{i L}}^{2}(t)=m_{0}^{2}+m_{e i}^{2}+\tilde{\alpha}_{G}\left[\frac{3}{2} \tilde{f}_{2}+\frac{3}{10} \tilde{f}_{1}\right] m_{1 / 2}^{2}+\left(-\frac{1}{2}+\sin ^{2} \theta_{W}\right) M_{Z}^{2} \cos (2 \beta), \\
m_{\tilde{e}_{i R}}^{2}(t)=m_{0}^{2}+m_{e i}^{2}+\frac{6}{5} \tilde{\alpha}_{G} \tilde{f}_{1} m_{1 / 2}^{2}-\sin ^{2} \theta_{W} M_{Z}^{2} \cos (2 \beta) .
\end{array}
$$


Using the above we find that

$$
\frac{1}{2}\left[m_{\tilde{d}_{i L}}^{2}(t)+m_{\tilde{d}_{i R}}^{2}(t)-\left(m_{\tilde{e}_{i L}}^{2}(t)+m_{\tilde{e}_{i R}}^{2}(t)\right)\right]=m_{d i}^{2}-m_{e i}^{2}+\tilde{\alpha}_{G}\left[\frac{8}{3} \gamma_{3}^{2} f_{3}-\frac{7}{15} \gamma_{1}^{2} f_{1}\right] .
$$

It is now easily seen that the last brace in Eq.(24) can vanish or even turn negative by appropriate choice of $\gamma_{3}$ vs $\gamma_{1}$. The above situation leads to a near equality of the squark and of the slepton masses discussed in the text of the paper. The $\mu$ parameter also has a strong dependence on nonuniversalities. This is seen by examining the relation that determines $\mu^{2}$, i.e.,

$$
\mu^{2}=\left(m_{H_{1}}^{2}-m_{H_{2}}^{2} \tan \beta^{2}\right)\left(\tan \beta^{2}-1\right)^{-1}-\frac{1}{2} M_{Z}^{2}+\Delta \mu^{2},
$$

where $\Delta \mu^{2}$ is the loop correction. Thus $m_{H_{1}}$, and $m_{H_{2}}$ are sensitive to nonuniversalities since

$$
m_{H_{1}}^{2}=m_{0}^{2}+\tilde{\alpha}_{G}\left(\frac{3}{2} \tilde{f}_{2}(t)+\frac{3}{10} \tilde{f}_{1}(t)\right) m_{1 / 2}^{2}
$$

where the nonuniversalities enter via the $\tilde{f}$ functions. Similarly $m_{H_{2}}$ is given by

$$
m_{H_{2}}^{2}=m_{1 / 2}^{2} \tilde{e}(t)+A_{0} m_{0} m_{1 / 2} \tilde{f}(t)+m_{0}^{2}\left(h(t)-k(t) A_{0}^{2}\right),
$$

where the tilde functions $\tilde{e}$ and $\tilde{f}$ are modified due to nonuniversality while the functions $h(t)$ and $k(t)$ are not unaffected (for definitions of these functions see the first paper of [15] and the references therein which also gives a more detailed discussion of this topic). The dependence on nonuniversalties is more complicated in this case because of the coupling with the top quark. Specifically, one can derive the following relation which gives the explicit dependence on nonuniversalities

$$
\frac{\partial \mu^{2}}{\partial \gamma_{a}}=\left(t^{2}-1\right)^{-1}\left(m_{1 / 2}^{2} g_{a}^{\prime}-t^{2}\left(m_{1 / 2}^{2} e_{a}^{\prime}+A_{0} m_{0} m_{1 / 2} f_{a}^{\prime}\right)\right)+\frac{\partial \Delta \mu^{2}}{\partial \gamma_{a}}
$$

where $g_{a}^{\prime}=\frac{\partial \tilde{g}}{\partial \gamma_{a}}, \tilde{g}=\tilde{\alpha}_{G}\left(\frac{3}{2} \tilde{f}_{2}+\frac{3}{10} \tilde{f}_{1}\right), e_{a}^{\prime}=\frac{\partial \tilde{e}}{\partial \gamma_{a}}$, and $f_{a}^{\prime}=\frac{\partial \tilde{f}}{\partial \gamma_{a}}$. One can make a semi-quantitative estimate of the dependence of $\mu^{2}$ on nonuniversalities from above. We note, however, that $\mu$ does not enter sensitively in the sum rule for the first two generation of squarks and sleptons and thus an estimate of the compression of the sfermion spectrum in the first two generations can be made without estimate of the $\mu$ parameter.

\section{Appendix C: Sample GNLSP benchmarks}

We provide here sample model points for the GNLSP class of models. Each model point obeys experimental constraints as discussed in the text. We use here SuSpect 2.41 coupled to MicrOMEGAs (MO) 2.2.CPC along with an independant code which agrees with $\mathrm{MO}$ at the pertuabtive level but accounts for the non-perturbative effects discussed in the text. Similar model points may be obtained with other spectrum calculators coupled to MO [see, for example, Table(4) where one such comparison is given, which, however, is only at the perturbative level]. 


\begin{tabular}{|l||c|c|c|c|c|c|}
\hline \hline GNSLP & $m_{0}(\mathrm{GeV})$ & $m_{1 / 2}(\mathrm{GeV})$ & $A_{0}(\mathrm{GeV})$ & $\tan \beta$ & $\delta_{2}$ & $\delta_{3}$ \\
\hline \hline GNSLP $_{\mathrm{A} 1}$ & 2949 & 692 & 3658 & 35 & 0.566 & -0.847 \\
GNSLP $_{\mathrm{A} 2}$ & 2706 & 783 & 4408 & 37 & 0.560 & -0.839 \\
\hline GNSLP $_{\mathrm{A} 3}$ & 2529 & 946 & 3873 & 41 & 0.560 & -0.837 \\
GNSLP $_{\mathrm{A} 4}$ & 2967 & 910 & 5114 & 27 & 0.557 & -0.834 \\
\hline GNSLP $_{\text {A5 }}$ & 2574 & 1058 & 4197 & 42 & 0.557 & -0.833 \\
GNSLP $_{\mathrm{A} 6}$ & 2821 & 1019 & 5050 & 20 & 0.554 & -0.830 \\
\hline GNSLP $_{\mathrm{A} 7}$ & 3008 & 1252 & -3241 & 27 & 0.558 & -0.836 \\
GNSLP $_{\mathrm{A} 8}$ & 2746 & 1265 & 5186 & 17 & 0.551 & -0.824 \\
\hline \hline
\end{tabular}

Table 6: GNLSP $_{\mathrm{A}}$ benchmarks: These models produce the correct relic density with SuSpect 2.41 coupled to MO 2.2. CPC including the non-perturbative corrections. Benchmarks here have $\left(\Omega h^{2}\right)_{\chi^{0}} \in(0.100,0.130)$. Here $m_{t}$ (pole)/ GeV $=170.9$ throughout.

\begin{tabular}{|c|c|c|c|c|c|c|c|c|c|}
\hline $\begin{array}{c}\text { GNSLP } \\
\text { Model }\end{array}$ & $\begin{array}{c}m_{h} \\
(\mathrm{GeV})\end{array}$ & $\begin{array}{c}m_{\widetilde{\chi}^{0}} \\
(\mathrm{GeV})\end{array}$ & $\begin{array}{c}m_{\tilde{g}} \\
(\mathrm{GeV})\end{array}$ & $\begin{array}{c}m_{\widetilde{\chi}_{1}^{ \pm}} \\
(\mathrm{GeV})\end{array}$ & $\begin{array}{c}m_{\widetilde{t}_{1}} \\
(\mathrm{GeV})\end{array}$ & \multicolumn{2}{|c|}{$\begin{array}{l}m_{A} \mid \tilde{h}_{1,2}^{\text {Frac }} \\
(\mathrm{GeV}) \mid-\end{array}$} & $\begin{array}{c}\sigma_{S I}\left(\widetilde{\chi}^{0} p\right) \\
(\mathrm{pb})\end{array}$ & $\begin{array}{c}\sigma_{S D}\left(\widetilde{\chi}^{0} p\right) \\
(\mathrm{pb})\end{array}$ \\
\hline GNSLP $_{\mathrm{A} 1}$ & 117 & 285 & 343 & 343 & 151560 & 2130 & 0.241 & $3.0 \times 10^{-8}$ & $8.0 \times 10^{-5}$ \\
\hline GNSLP $_{\mathrm{A} 2}$ & 117 & 337 & 387 & 869 & 1225 & 1952 & 0.004 & $2.7 \times 10^{-10}$ & $2.8 \times 10^{-7}$ \\
\hline GNSLP $_{\mathrm{A} 3}$ & 116 & 398 & 456 & 480 & 1190 & 1540 & 0.135 & $2.3 \times 10^{-8}$ & $2.4 \times 10^{-5}$ \\
\hline GNSLP $_{\mathrm{A} 4}$ & 117 & 399 & 454 & 1080 & 1276 & 2724 & 0.003 & $1.3 \times 10^{-10}$ & $1.1 \times 10^{-7}$ \\
\hline GNSLP $_{\mathrm{A} 5}$ & 116 & 447 & 510 & 531 & 1161 & 1514 & 0.131 & $2.2 \times 10^{-8}$ & $1.9 \times 10^{-5}$ \\
\hline GNSLP $_{\mathrm{A} 6}$ & 117 & 448 & 507 & 1064 & 1149 & 2886 & 0.003 & $2.1 \times 10^{-10}$ & $1.4 \times 10^{-7}$ \\
\hline GNSLP $_{\mathrm{A} 7}$ & 120 & 551 & 618 & 645 & 1332 & 2718 & 0.108 & $1.3 \times 10^{-8}$ & $1.0 \times 10^{-5}$ \\
\hline GNSLP $_{\mathrm{A} 8}$ & 116 & 557 & 624 & 970 & 1032 & 2960 & 0.007 & $6.7 \times 10^{-10}$ & $3.3 \times 10^{-7}$ \\
\hline
\end{tabular}

Table 7: GNLSP $_{\mathrm{A}}$ properties: The variation in the spin independent cross section over two orders of magnitude arises due to variations in the higgsino vs the bino component of the LSP. The higgsino fraction has been defined by $\tilde{h}_{1,2}^{\text {Frac }}=|\gamma|^{2}+|\delta|^{2}$, where the normalized LSP mass eigenstate is $\tilde{\chi}^{0}=\alpha \tilde{b}+\beta \tilde{w}+\gamma \tilde{h}_{1}+\delta \tilde{h}_{2}$ as in the notation defined in Sec.(44). 


\begin{tabular}{|c||c|c|c|c|c|c|}
\hline \hline GNSLP & $m_{0}(\mathrm{GeV})$ & $m_{1 / 2}(\mathrm{GeV})$ & $A_{0}(\mathrm{GeV})$ & $\tan \beta$ & $\delta_{2}$ & $\delta_{3}$ \\
\hline \hline GNSLP $_{\mathrm{B} 1}$ & 2421 & 736 & 3414 & 51 & 0.000 & -0.841 \\
GNSLP $_{\mathrm{B} 2}$ & 3406 & 734 & 4655 & 35 & 0.000 & -0.848 \\
\hline GNSLP $_{\mathrm{B} 3}$ & 2890 & 945 & -2977 & 47 & 0.000 & -0.844 \\
GNSLP $_{\mathrm{B} 4}$ & 3772 & 988 & 5894 & 46 & 0.000 & -0.837 \\
\hline GNSLP $_{\mathrm{B} 5}$ & 2857 & 1158 & -2631 & 24 & 0.000 & -0.842 \\
GNSLP $_{\mathrm{B} 6}$ & 2943 & 1142 & 5006 & 12 & 0.000 & -0.831 \\
\hline GNSLP $_{\mathrm{B} 7}$ & 3188 & 1376 & -2479 & 7 & 0.000 & -0.837 \\
GNSLP $_{\mathrm{B} 8}$ & 2659 & 1380 & 5028 & 37 & 0.000 & -0.825 \\
\hline \hline
\end{tabular}

Table 8: GNLSP G $_{\mathrm{B}}$ benchmarks: As in Table(6) the displayed models produce the correct relic density. Benchmarks here have $\left(\Omega h^{2}\right)_{\chi^{0}} \in(0.100,0.120)$.

\begin{tabular}{|l||c|c|c|c|c|c|c|c|}
\hline \hline $\begin{array}{l}\text { GNSLP } \\
\text { Model }\end{array}$ & $\begin{array}{c}m_{h} \\
(\mathrm{GeV})\end{array}$ & $\begin{array}{c}m_{\tilde{\chi}^{0}} \\
(\mathrm{GeV})\end{array}$ & $\begin{array}{c}m_{\tilde{g}} \\
(\mathrm{GeV})\end{array}$ & $\begin{array}{c}m_{\tilde{\chi}_{1}^{ \pm}} \\
(\mathrm{GeV})\end{array}$ & $\begin{array}{c}m_{\tilde{t}_{1}} \\
(\mathrm{GeV})\end{array}$ & $\begin{array}{c}m_{A} \mid \tilde{h}_{1,2}^{\text {Frac }} \\
(\mathrm{GeV}) \mid-\end{array}$ & $\begin{array}{c}\sigma_{S I}\left(\tilde{\chi}^{0} p\right) \\
(\mathrm{pb})\end{array}$ & $\begin{array}{c}\sigma_{S D}\left(\widetilde{\chi}^{0} p\right) \\
(\mathrm{pb})\end{array}$ \\
\hline \hline GNSLP $_{\mathrm{B} 1}$ & 116 & 313 & 364 & 557 & 1223 & $409 \mid 0.009$ & $2.4 \times 10^{-8}$ & $1.2 \times 10^{-6}$ \\
GNSLP $_{\mathrm{B} 2}$ & 119 & 316 & 364 & 576 & 1734 & $2520 \mid 0.003$ & $1.4 \times 10^{-10}$ & $2.0 \times 10^{-7}$ \\
\hline GNSLP $_{\mathrm{B} 3}$ & 118 & 417 & 484 & 582 & 1507 & $665 \mid 0.035$ & $2.0 \times 10^{-8}$ & $5.2 \times 10^{-6}$ \\
GNSLP $_{\mathrm{B} 4}$ & 119 & 428 & 488 & 779 & 1818 & $1756 \mid 0.002$ & $1.0 \times 10^{-10}$ & $5.6 \times 10^{-8}$ \\
\hline GNSLP $_{\mathrm{B} 5}$ & 119 & 502 & 566 & 583 & 1475 & $2610 \mid 0.140$ & $1.8 \times 10^{-8}$ & $1.7 \times 10^{-5}$ \\
GNSLP $_{\mathrm{B} 6}$ & 117 & 502 & 565 & 948 & 1299 & $3177 \mid 0.002$ & $1.7 \times 10^{-10}$ & $6.8 \times 10^{-8}$ \\
\hline GNSLP $_{\mathrm{B} 7}$ & 117 & 601 & 672 & 701 & 1676 & $3382 \mid 0.113$ & $1.8 \times 10^{-8}$ & $7.4 \times 10^{-6}$ \\
GNSLP $_{\mathrm{B} 8}$ & 116 & 598 & 669 & 1051 & 1121 & $2031 \mid 0.004$ & $4.3 \times 10^{-10}$ & $1.6 \times 10^{-7}$ \\
\hline \hline
\end{tabular}

Table 9: GNLSP $_{\mathrm{B}}$ properties: The table gives an analysis similar to that of Table(7) for GNLSP $_{\mathrm{B}}$ models. 


\begin{tabular}{|l||c|c|c|c|c|c|}
\hline \hline GNSLP & $m_{0}(\mathrm{GeV})$ & $m_{1 / 2}(\mathrm{GeV})$ & $A_{0}(\mathrm{GeV})$ & $\tan \beta$ & $\delta_{2}$ & $\delta_{3}$ \\
\hline \hline GNSLP $_{\mathrm{C} 1}$ & 1604 & 450 & 2035 & 49 & -0.317 & -0.852 \\
GNSLP $_{\mathrm{C} 2}$ & 2119 & 696 & 2860 & 44 & 0.291 & -0.845 \\
GNSLP $_{\mathrm{C} 3}$ & 2443 & 948 & 2823 & 11 & -0.227 & -0.842 \\
GNSLP $_{\mathrm{C} 4}$ & 3850 & 1111 & 4388 & 9 & 0.209 & -0.840 \\
GNSLP $_{\mathrm{C} 5}$ & 2599 & 1270 & 3656 & 14 & -0.009 & -0.833 \\
GNSLP $_{\mathrm{C} 6}$ & 2458 & 1479 & 5045 & 35 & 0.462 & -0.823 \\
\hline GNSLP $_{\mathrm{C} 7}$ & 2087 & 453 & 2359 & 21 & 0.292 & -0.862 \\
GNSLP $_{\mathrm{C} 8}$ & 1958 & 674 & 2950 & 22 & 0.299 & -0.843 \\
GNSLP $_{\mathrm{C} 9}$ & 3874 & 1098 & 4455 & 8 & -0.018 & -0.839 \\
GNSLP $_{\mathrm{C} 10}$ & 2543 & 1337 & 4188 & 49 & -0.147 & -0.826 \\
GNSLP $_{\mathrm{C} 11}$ & 3288 & 1431 & 5879 & 15 & 0.703 & -0.822 \\
GNSLP $_{\mathrm{C} 12}$ & 3942 & 1755 & 5995 & 36 & 0.080 & -0.825 \\
\hline \hline
\end{tabular}

Table 10: GNLSP $_{\mathrm{C}}$ benchmarks: A sample of benchmarks in GNLSP $_{\mathrm{C}}$ in a random distribution in $\delta_{2}$ and $\delta_{3}$. Many of the models listed above have a substantial higgsino component and part of the parameter space would be accessible to current and future experiments for the direct detection of dark matter. Benchmarks here have $\left(\Omega h^{2}\right)_{\chi^{0}} \in(0.100,0.138)$.

\begin{tabular}{|l||c|c|c|c|c|c|c|c|}
\hline \hline $\begin{array}{l}\text { GNSLP } \\
\text { Model }\end{array}$ & $\begin{array}{c}m_{h} \\
(\mathrm{GeV})\end{array}$ & $\begin{array}{c}m_{\tilde{\chi}^{0}} \\
(\mathrm{GeV})\end{array}$ & $\begin{array}{c}m_{\tilde{g}} \\
(\mathrm{GeV})\end{array}$ & $\begin{array}{c}m_{\tilde{\chi}_{1}^{ \pm}} \\
(\mathrm{GeV})\end{array}$ & $\begin{array}{c}m_{\widetilde{t}_{1}} \\
(\mathrm{GeV})\end{array}$ & $\begin{array}{c}m_{A} \mid \tilde{h}_{1,2}^{\text {Frac }} \\
(\mathrm{GeV}) \mid-\end{array}$ & $\begin{array}{c}\sigma_{S I}\left(\tilde{\chi}^{0} p\right) \\
(\mathrm{pb})\end{array}$ & $\begin{array}{c}\sigma_{S D}\left(\tilde{\chi}^{0} p\right) \\
(\mathrm{pb})\end{array}$ \\
\hline \hline GNSLP $_{\mathrm{C} 1}$ & 113 & 185 & 227 & 228 & 830 & $516 \mid 0.022$ & $3.6 \times 10^{-8}$ & $9.0 \times 10^{-6}$ \\
GNSLP $_{\mathrm{C} 2}$ & 115 & 291 & 338 & 423 & 1073 & $1065 \mid 0.056$ & $1.4 \times 10^{-8}$ & $1.6 \times 10^{-5}$ \\
GNSLP $_{\mathrm{C} 3}$ & 115 & 404 & 461 & 508 & 1315 & $2488 \mid 0.075$ & $1.3 \times 10^{-8}$ & $1.2 \times 10^{-5}$ \\
$\mathrm{GNSLP}_{\mathrm{C} 4}$ & 117 & 481 & 546 & 587 & 2078 & $3939 \mid 0.098$ & $1.4 \times 10^{-8}$ & $1.0 \times 10^{-5}$ \\
$\mathrm{GNSLP}_{\mathrm{C} 5}$ & 116 & 542 & 610 & 609 & 1320 & $2677 \mid 0.200$ & $2.9 \times 10^{-8}$ & $1.9 \times 10^{-5}$ \\
GNSLP $_{\mathrm{C} 6}$ & 115 & 632 & 704 & 716 & 830 & $2042 \mid 0.130$ & $2.0 \times 10^{-8}$ & $9.7 \times 10^{-6}$ \\
\hline GNSLP $_{\mathrm{C} 7}$ & 115 & 189 & 223 & 333 & 1114 & $1938 \mid 0.047$ & $5.1 \times 10^{-9}$ & $2.2 \times 10^{-5}$ \\
GNSLP $_{\mathrm{C} 8}$ & 114 & 289 & 333 & 581 & 924 & $1887 \mid 0.012$ & $1.2 \times 10^{-9}$ & $1.8 \times 10^{-6}$ \\
GNSLP $_{\mathrm{C} 9}$ & 117 & 483 & 546 & 816 & 2088 & $3996 \mid 0.010$ & $1.1 \times 10^{-9}$ & $6.0 \times 10^{-7}$ \\
GNSLP $_{\mathrm{C} 10}$ & 116 & 576 & 648 & 797 & 1242 & $956 \mid 0.018$ & $5.4 \times 10^{-9}$ & $1.3 \times 10^{-6}$ \\
GNSLP $_{\mathrm{C} 11}$ & 117 & 634 & 708 & 909 & 1310 & $3558 \mid 0.015$ & $1.7 \times 10^{-9}$ & $8.0 \times 10^{-7}$ \\
GNSLP $_{\mathrm{C} 12}$ & 119 & 769 & 849 & 948 & 1965 & $2909 \mid 0.031$ & $3.7 \times 10^{-9}$ & $1.6 \times 10^{-6}$ \\
\hline \hline
\end{tabular}

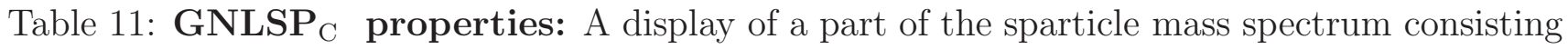
of light Higgs and CP odd Higgs masses, and masses of the LSP, NLSP, light chargino, and light stop along with spin independent and dependent cross sections. Models shown here include those with higgsino like LSPs and as well as those with mixed higgsino and bino LSPs, and LSPs which are mostly bino. The horizontal line emphasizes separation of the $\sigma_{S I} / \mathrm{pb}$ from $O\left(10^{-8}\right)$ to $O\left(10^{-9}\right)$. 


\section{References}

[1] D. Feldman, Z. Liu and P. Nath, Phys. Rev. Lett. 99, 251802 (2007).

[2] D. N. Spergel et al. [WMAP Collaboration], Astrophys. J. Suppl. 170, 377 (2007); E. Komatsu et al. [WMAP Collaboration], Astrophys. J. Suppl. 180, 330 (2009).

[3] D. Feldman, Z. Liu and P. Nath, Phys. Lett. B 662, 190 (2008).

[4] D. Feldman, Z. Liu and P. Nath, JHEP 0804, 054 (2008).

[5] S. Profumo and C. E. Yaguna, Phys. Rev. D 69, 115009 (2004); Phys. Rev. D 70, 095004 (2004) S. Profumo, Phys. Rev. D 72, 103521 (2005).

[6] N. Bernal, A. Djouadi and P. Slavich, JHEP 0707, 016 (2007).

[7] I. Gogoladze, R. Khalid and Q. Shafi, arXiv:0903.5204 [hep-ph].

[8] H. Baer, K. m. Cheung and J. F. Gunion, Phys. Rev. D 59, 075002 (1999); S. Raby and K. Tobe, Nucl. Phys. B 539, 3 (1999).

[9] H. Baer, A. Mustafayev, E. K. Park, S. Profumo and X. Tata, JHEP 0604, 041 (2006).

[10] P. Nath and R. Arnowitt, Phys. Rev. D 56, 2820 (1997); J. R. Ellis, K. A. Olive and Y. Santoso, Phys. Lett. B 539, 107 (2002) [arXiv:hep-ph/0204192]; H. Baer, A. Mustafayev, S. Profumo, A. Belyaev and X. Tata, JHEP 0507, 065 (2005) [arXiv:hep-ph/0504001; U. Chattopadhyay and D. Das, arXiv:0809.4065 [hep-ph]. See [3, 4, 14, 15, 16] for further references specifically on the nonuniversality of the gaugino masses.

[11] A. H. Chamseddine, R. Arnowitt and P. Nath, Phys. Rev. Lett. 49 (1982) 970; P. Nath, R. L. Arnowitt and A. H. Chamseddine, Nucl. Phys. B 227, 121 (1983).

[12] L. Hall, J. Lykken and S. Weinberg, Phys. Rev. D27, 2359 (1983); For historical reviews see: H. P. Nilles, Phys. Rept. 110, 1 (1984); P. Nath, arXiv:hep-ph/0307123.

[13] A. Arvanitaki, C. Davis, P. W. Graham, A. Pierce and J. G. Wacker, Phys. Rev. D 72, 075011 (2005); A. Arvanitaki, S. Dimopoulos, A. Pierce, S. Rajendran and J. G. Wacker, Phys. Rev. D 76, 055007 (2007) arXiv:hep-ph/0506242]; A. Alves, O. Eboli and T. Plehn, Phys. Rev. D 74, 095010 (2006); J. Alwall, M. P. Le, M. Lisanti and J. G. Wacker, Phys. Lett. B 666, 34 (2008); Phys. Rev. D 79, 015005 (2009); J. Alwall, S. de Visscher and F. Maltoni, JHEP 0902, 017 (2009); G. L. Kane, A. A. Petrov, J. Shao and L. T. Wang, arXiv:0805.1397 [hep-ph]. B. S. Acharya, P. Grajek, G. L. Kane, E. Kuflik, K. Suruliz and L. T. Wang, arXiv:0901.3367 [hep-ph].

[14] J.R. Ellis, K. Enqvist, D.V. Nanopoulos and K. Tamvakis, Phys. Lett. B 155, 381 (1985); M. Drees, Phys. Lett. B 158, 409 (1985); G. Anderson, C.H. Chen, J.F. Gunion, J.D. Lykken, T. Moroi and Y. Yamada, [hep-ph/9609457]. 
[15] A. Corsetti and P. Nath, Phys. Rev. D 64, 125010 (2001); U. Chattopadhyay and P. Nath, Phys. Rev. D 65, 075009 (2002); A. Birkedal-Hansen and B. D. Nelson, Phys. Rev. D 67, 095006 (2003); U. Chattopadhyay and D. P. Roy, Phys. Rev. D 68, 033010 (2003); D. G. Cerdeno and C. Munoz, JHEP 0410, 015 (2004); G. Belanger, F. Boudjema, A. Cottrant, A. Pukhov and A. Semenov, Nucl. Phys. B 706, 411 (2005); H. Baer, A. Mustafayev, E. K. Park, S. Profumo and X. Tata, JHEP 0604, 041 (2006); K. Choi and H. P. Nilles JHEP 0704 (2007) 006; I. Gogoladze, R. Khalid, N. Okada and Q. Shafi, arXiv:0811.1187 [hep-ph]; S. Bhattacharya, A. Datta and B. Mukhopadhyaya, Phys. Rev. D 78, 115018 (2008); M. E. Gomez, S. Lola, P. Naranjo and J. Rodriguez-Quintero, arXiv:0901.4013 [hepph]; B. Altunkaynak, P. Grajek, M. Holmes, G. Kane and B. D. Nelson, arXiv:0901.1145 [hep-ph]; U. Chattopadhyay, D. Das and D. P. Roy, arXiv:0902.4568 [hep-ph]; S. Bhattacharya and J. Chakrabortty, arXiv:0903.4196 [hep-ph].

[16] S. P. Martin, arXiv:0903.3568 [hep-ph].

[17] A. Djouadi, J. L. Kneur and G. Moultaka, Comput. Phys. Commun. 176, 426 (2007); Present version 2.41 (2008).

[18] B. C. Allanach, Comput. Phys. Commun. 143, 305 (2002) arXiv:hep-ph/0104145; Present version 3.02 (2009).

[19] W. Porod, Comput. Phys. Commun. 153, 275 (2003); Present version 2.23 (2005).

[20] G. Belanger, F. Boudjema, A. Pukhov and A. Semenov, Comput. Phys. Commun. 176, 367 (2007); G. Belanger, F. Boudjema, A. Pukhov and A. Semenov, Comput. Phys. Commun. 174, 577 (2006); G. Belanger, F. Boudjema, A. Pukhov and A. Semenov, Comput. Phys. Commun. 149 (2002) 103; Present version 2.2 CPC.

[21] A. Pukhov, arXiv:hep-ph/0412191.

[22] E. Komatsu et al. [WMAP Collaboration], Astrophys. J. Suppl. 180, 330 (2009).

[23] G. Degrassi, P. Gambino and G. F. Giudice, JHEP 0012 (2000) 009; D. A. Demir and K. A. Olive, Phys. Rev. D 65, 034007 (2002) arXiv:hep-ph/0107329]; A. J. Buras et.al., Nucl. Phys. B 659 (2003) 3; M. E. Gomez, T. Ibrahim, P. Nath and S. Skadhauge, Phys. Rev. D 74 (2006) 015015; G. Degrassi, P. Gambino and P. Slavich, Phys. Lett. B 635 (2006) 335.

[24] E. Barberio et al. [Heavy Flavor Averaging Group], arXiv:0808.1297 [hep-ex].

[25] M. Misiak et al., Phys. Rev. Lett. 98 (2007) 022002.

[26] S. R. Choudhury and N. Gaur, Phys. Lett. B 451, 86 (1999); K. S. Babu and C. F. Kolda, Phys. Rev. Lett. 84, 228 (2000) [arXiv:hep-ph/9909476]; C. Bobeth, T. Ewerth, F. Kruger and J. Urban, Phys. Rev. D 64, 074014 (2001); R. L. Arnowitt, B. Dutta, T. Kamon and M. Tanaka, Phys. Lett. B 538, 121 (2002) [arXiv:hep-ph/0203069]; A. Dedes, H. K. Dreiner, U. Nierste and P. Richardson, arXiv:hep-ph/0207026. J. K. Mizukoshi, 
X. Tata and Y. Wang, Phys. Rev. D 66, 115003 (2002) [arXiv:hep-ph/0208078]. T. Ibrahim and P. Nath, Phys. Rev. D 67, 016005 (2003) arXiv:hep-ph/0208142.

[27] T. Aaltonen et al. [CDF Collaboration], Phys. Rev. Lett. 100, 101802 (2008) arXiv:0712.1708 [hep-ex]].

[28] A. Djouadi, M. Drees and J. L. Kneur, JHEP 0603, 033 (2006).

[29] G. Abbiendi et al. [OPAL Collaboration], Eur. Phys. J. C 35, 1 (2004).

[30] R. Barate et al. [LEP Working Group for Higgs boson searches], Phys. Lett. B 565, 61 (2003); The ALEPH, DELPHI, L3 and OPAL Collaborations, LHWG-Note 2005-01.

[31] V. M. Abazov et al. [D0 Collaboration], Phys. Lett. B 660, 449 (2008).

[32] T. Aaltonen et al. [CDF Collaboration], arXiv:0811.2512 [hep-ex].

[33] A. Belyaev, S. Dar, I. Gogoladze, A. Mustafayev and Q. Shafi, arXiv:0712.1049 [hep-ph].

[34] R. L. Arnowitt, B. Dutta, A. Gurrola, T. Kamon, A. Krislock and D. Toback, Phys. Rev. Lett. 100 (2008) 231802; R. L. Arnowitt et al., Phys. Lett. B 649, 73 (2007).

[35] D. Feldman, Z. Liu and P. Nath, AIP Conf. Proc. 1078, 116 (2009).

[36] N. Bhattacharyya, A. Datta and S. Poddar, Phys. Rev. D 78, 075030 (2008).

[37] D. Feldman, Z. Liu and P. Nath, Phys. Rev. D 78, 083523 (2008).

[38] S. Biswas and B. Mukhopadhyaya, arXiv:0902.4349 [hep-ph].

[39] K. Griest and D. Seckel, Phys. Rev. D 43, 3191 (1991).

[40] K. L. Chan, U. Chattopadhyay and P. Nath, Phys. Rev. D 58, 096004 (1998); J. L. Feng, K. T. Matchev and T. Moroi, Phys. Rev. Lett. 84, 2322 (2000); U. Chattopadhyay, A. Corsetti and P. Nath, Phys. Rev. D 68, 035005 (2003); H. Baer, C. Balazs, A. Belyaev, T. Krupovnickas and X. Tata, JHEP 0306, 054 (2003).

[41] S. P. Martin and P. Ramond, Phys. Rev. D 48, 5365 (1993) arXiv:hep-ph/9306314.

[42] H. Baer, V. Barger, G. Shaughnessy, H. Summy and L. t. Wang, Phys. Rev. D 75, 095010 (2007).

[43] S. P. Martin, Phys. Rev. D 75, 115005 (2007).

[44] G. L. Kane and J. P. Leveille, Phys. Lett. B 112, 227 (1982); P. R. Harrison and C. H. Llewellyn Smith, Nucl. Phys. B 213, 223 (1983); E. Reya and D. P. Roy, Phys. Lett. B 141, 442 (1984); S. Dawson, E. Eichten and C. Quigg, Phys. Rev. D 31, 1581 (1985). 
[45] W. Beenakker, R. Hopker, M. Spira and P. M. Zerwas, Nucl. Phys. B 492, 51 (1997).

[46] This is MSEL=39 in [49].

[47] A. Djouadi, M. M. Muhlleitner and M. Spira, Acta Phys. Polon. B 38, 635 (2007); M. Muhlleitner, A. Djouadi and Y. Mambrini, Comput. Phys. Commun. 168, 46 (2005); A. Djouadi, J. Kalinowski and M. Spira, Comput. Phys. Commun. 108, 56 (1998).

[48] F. E. Paige, S. D. Protopopescu, H. Baer and X. Tata, arXiv:hep-ph/0312045.

[49] T. Sjostrand, S. Mrenna, P. Skands, JHEP 0605, 026 (2006).

[50] W. Beenakker, M. Klasen, M. Kramer, T. Plehn, M. Spira and P. M. Zerwas, Phys. Rev. Lett. 83, 3780 (1999); W. Beenakker, R. Hopker and M. Spira, arXiv:hep-ph/9611232.

[51] P. Skands et al., JHEP 0407, 036 (2004).

[52] PGS-4; J. Conway et. al, CDF : This code simulates LHC detector effects, with a tracking system, EM and hadronic calorimetry, and a muon system, taking events simulated with PYTHIA [49] and reconstructs photons, electrons/muons, hadronically decaying taus, and hadronic jets.

[53] G. L. Bayatian et al. [CMS Collaboration], J. Phys. G 34, 995 (2007).

[54] H. E. Haber and G. L. Kane, Nucl. Phys. B 232, 333 (1984); E. Ma and G. G. Wong, Mod. Phys. Lett. A 3, 1561 (1988); H. Baer, R. M. Barnett, M. Drees, J. F. Gunion, H. E. Haber, D. L. Karatas and X. R. Tata, Int. J. Mod. Phys. A 2, 1131 (1987); H. Baer, X. Tata and J. Woodside, Phys. Rev. D 42, 1568 (1990).

[55] M. Toharia and J. D. Wells, JHEP 0602, 015 (2006).

[56] H. Baer, R. M. Barnett, M. Drees, J. F. Gunion, H. E. Haber, D. L. Karatas and X. R. Tata, Int. J. Mod. Phys. A 2, 1131 (1987).

[57] R. H. K. Kadala, P. G. Mercadante, J. K. Mizukoshi and X. Tata, Eur. Phys. J. C 56, 511 (2008).

[58] V. N. Lebedenko et al., arXiv:0812.1150 [astro-ph].

[59] Z. Ahmed et al. [CDMS Collaboration], Phys. Rev. Lett. 102, 011301 (2009).

[60] J. Angle et al. [XENON Collaboration], Phys. Rev. Lett. 100, 021303 (2008).

[61] See R. Gaitskell, J. Filippini, et al. http://dendera.berkeley.edu/plotter/entryform.html Based on : SuperCDMS (Projected) 2-ST@Soudan,SuperCDMS Proposal, SuperCDMS (Projected) 25kg (7-ST@Snolab),SuperCDMS Proposal, LUX Proposal, 300 kg LXe Projection.

[62] O. Adriani et al. [PAMELA Collaboration], Nature 458, 607 (2009). 